Prepared in cooperation with the Albuquerque Bernalillo County Water Utility Authority

\title{
Water-Level Data for the Albuquerque Basin and Adjacent Areas, Central New Mexico, Period of Record Through September 30, 2019
}

Data Series 1129 



\section{Water-Level Data for the Albuquerque Basin and Adjacent Areas, Central New Mexico, Period of Record Through September 30, 2019}

By Joseph E. Beman

Prepared in cooperation with the Albuquerque Bernalillo County Water Utility Authority

Data Series 1129 


\title{
U.S. Department of the Interior \\ DAVID BERNHARDT, Secretary
}

\author{
U.S. Geological Survey \\ James F. Reilly II, Director
}

U.S. Geological Survey, Reston, Virginia: 2020

For more information on the USGS - the Federal source for science about the Earth, its natural and living resources, natural hazards, and the environment—visit http://www.usgs.gov or call 1-888-ASK-USGS.

For an overview of USGS information products, including maps, imagery, and publications, visit http://www.usgs.gov/pubprod.

Any use of trade, firm, or product names is for descriptive purposes only and does not imply endorsement by the U.S. Government.

Although this information product, for the most part, is in the public domain, it also may contain copyrighted materials as noted in the text. Permission to reproduce copyrighted items must be secured from the copyright owner.

Suggested citation:

Beman, J.E., 2020, Water-level data for the Albuquerque Basin and adjacent areas, central New Mexico, period of record through September 30, 2019: U.S. Geological Survey Data Series 1129, 40 p., https://doi.org/10.3133/ds1129.

ISSN 2327-638X (online) 


\section{Contents}

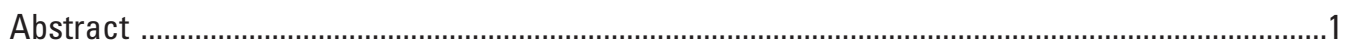

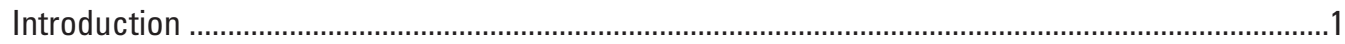

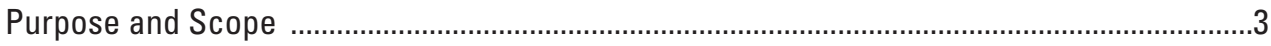

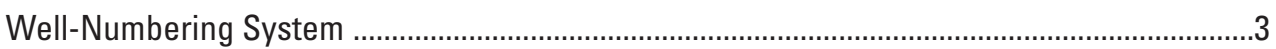

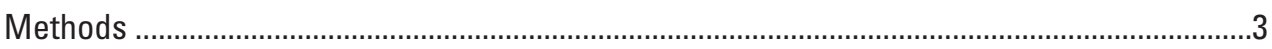

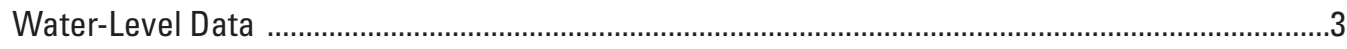

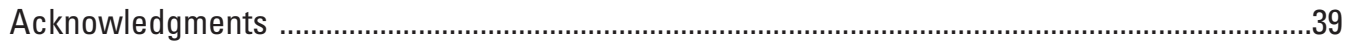

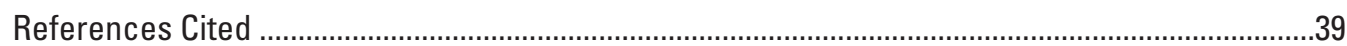

\section{Figures}

1. Map showing location of the study area and selected monitoring wells and piezometers in and adjacent to the Albuquerque Basin, central New Mexico ................2

2. Map showing location of selected monitoring wells and piezometers within the Albuquerque, New Mexico, metropolitan area ............................................................9

3. Diagram showing system for numbering wells and piezometers in New Mexico .........10

4. Graphs showing water-level data for selected wells and piezometers in and adjacent to the Albuquerque Basin, central New Mexico, period of record through September 30, 2019

\section{Tables}

1. Data for selected wells and piezometers in and adjacent to the Albuquerque Basin, central New Mexico

\section{Conversion Factors}

\begin{tabular}{|c|c|c|}
\hline Multiply & By & To obtain \\
\hline \multicolumn{3}{|c|}{ Length } \\
\hline foot (ft) & 0.3048 & meter (m) \\
\hline mile (mi) & 1.609 & kilometer (km) \\
\hline \multicolumn{3}{|c|}{ Area } \\
\hline acre & 4,047 & square meter $\left(\mathrm{m}^{2}\right)$ \\
\hline
\end{tabular}

Vertical coordinate information is referenced to the North American Vertical Datum of 1988 (NAVD 88).

Water year is defined as beginning on 0 ctober 1 and continuing through September 30 of the following year and designated by the calendar year in which it ends. 



\title{
Water-Level Data for the Albuquerque Basin and Adjacent Areas, Central New Mexico, Period of Record Through September 30, 2019
}

\author{
By Joseph E. Beman
}

\section{Abstract}

The Albuquerque Basin, located in central New Mexico, is about 100 miles long and 25-40 miles wide. The basin is hydrologically defined as the extent of consolidated and unconsolidated deposits of Tertiary and Quaternary age that encompasses the structural Rio Grande Rift between San Acacia to the south and Cochiti Lake to the north. A 20-percent population increase in the basin from 1990 to 2000 and a 22-percent population increase from 2000 to 2010 resulted in an increased demand for water in areas within the basin. Drinking-water supplies throughout the basin were obtained solely from groundwater resources until December 2008, when the Albuquerque Bernalillo County Water Utility Authority (ABCWUA) began treatment and distribution of surface water from the Rio Grande through the San Juan-Chama Drinking Water Project.

An initial network of wells was established by the U.S. Geological Survey (USGS) in cooperation with the City of Albuquerque from April 1982 through September 1983 to monitor changes in groundwater levels throughout the Albuquerque Basin. In 1983, this network consisted of 6 wells with analog-to-digital recorders and 27 wells where water levels were measured monthly. As of 2019, the network consisted of 120 wells and piezometers. (A piezometer is a specialized well open to a specific depth in the aquifer, often of small diameter and nested with other piezometers screened at different depths.) The USGS, in cooperation with the ABCWUA, the New Mexico Office of the State Engineer, and Bernalillo County, measures water levels from the 120 wells and piezometers in the network; this report, prepared in cooperation with the ABCWUA, presents water-level data collected by USGS personnel at those 120 sites through water year 2019 (October 1, 2018, through September 30, 2019). Water levels that were collected from those discontinued wells in previous water years were published in previous USGS reports.

\section{Introduction}

The Albuquerque Basin, located in central New Mexico, is about 100 miles long and 25-40 miles wide (fig. 1). The basin is defined as the extent of consolidated and unconsolidated deposits of Tertiary and Quaternary age that encompasses the structural Rio Grande Rift (Thorn and others, 1993). The basin is approximately bisected by the southward-flowing Rio Grande, the only perennial stream extending through the length of it. The study area, which includes the Albuquerque Basin and adjacent areas, extends from just upstream of Cochiti Lake south to San Acacia and from near Tijeras Canyon west to near the intersection of Interstate 40 and the Bernalillo-Cibola County line.

In 2000, the population of the Albuquerque Basin was about 690,000 (Bartolino and Cole, 2002). According to 2010 U.S. Census Bureau data, the 2010 population was about 840,000 (U.S. Census Bureau, 2019; calculated as the sum of population for census tract centers within the basin). The basin population increased about 20 percent from 1990 to 2000 (Thorn and others, 1993; Bartolino and Cole, 2002) and about 22 percent from 2000 to 2010 (U.S. Census Bureau, 2019). The majority of the population is concentrated within the city limits of Albuquerque, which had a population of 448,607 in 2000 and 545,852 in 2010 (U.S. Census Bureau, 2012). Prior to 2008, demand for groundwater increased as population increased because drinking-water supplies throughout the Albuquerque Basin were obtained solely from groundwater sources. In December 2008, the Albuquerque Bernalillo County Water Utility Authority (ABCWUA) began treatment and distribution of surface water from the Rio Grande to their customers through the San Juan-Chama Drinking Water Project. 


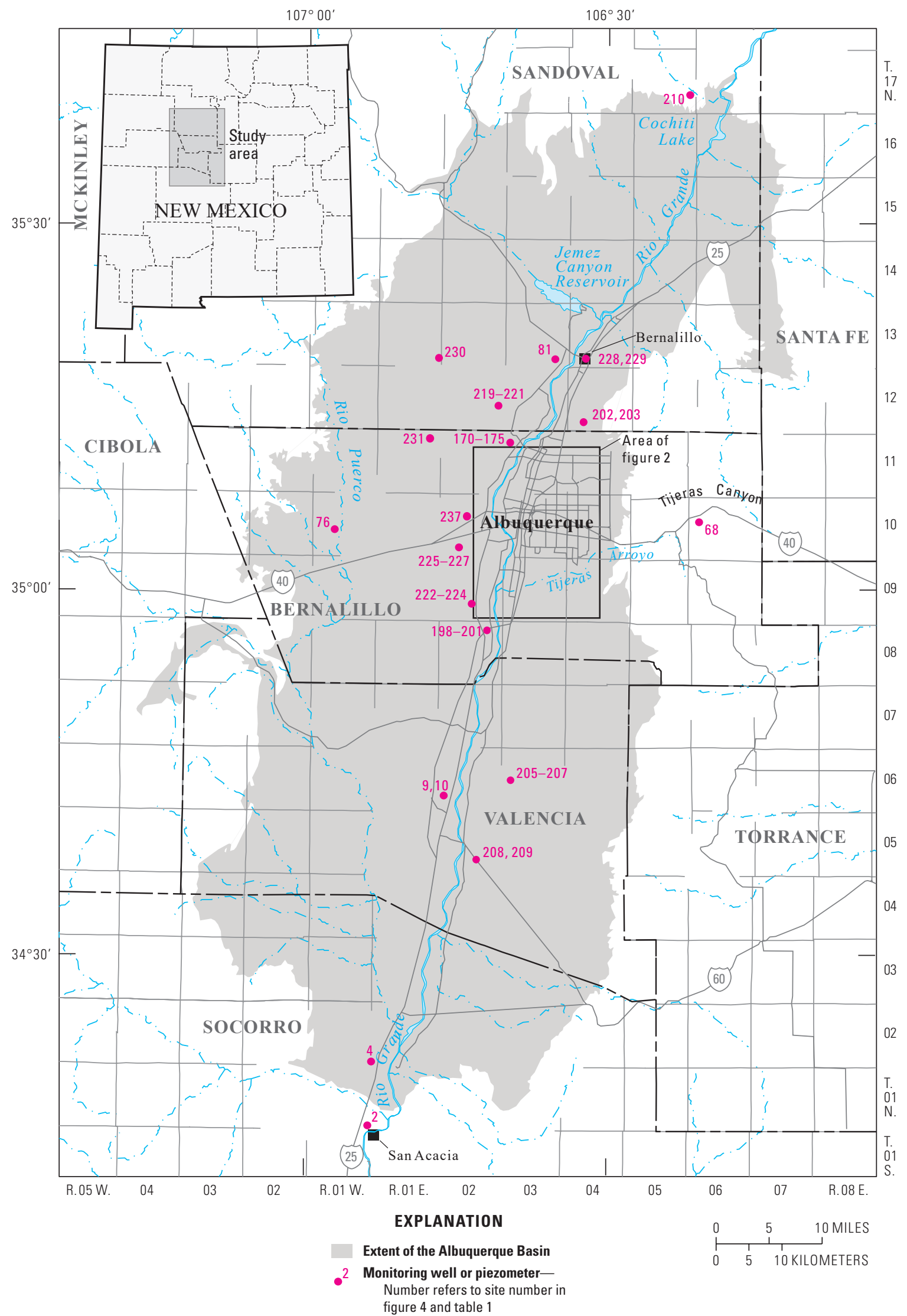

Figure 1. Location of the study area and selected monitoring wells and piezometers in and adjacent to the Albuquerque Basin, central New Mexico. 
An initial network of wells was established by the U.S. Geological Survey (USGS) in cooperation with the City of Albuquerque from April 1982 through September 1983 to monitor changes in groundwater levels throughout the Albuquerque Basin. In 1983, this network consisted of 6 wells with analog-to-digital recorders and 27 wells where water levels were measured monthly. Since the initial installation, additional wells and piezometers have been added to the network, and as of 2019 the network consisted of 120 wells and piezometers (table 1). (A piezometer is a specialized well screened at a specific depth in the aquifer, often of small diameter and nested with other piezometers screened at different depths.) Of these wells and piezometers, 66 are equipped with continuously recording data loggers, and 54 are measured discretely (semiannually, quarterly, or irregularly) with a steel or electric tape. Discrete measurements are also collected at sites with data loggers but are not shown in this report with the exception of sites 42-45 (table 1), which have more than 10 years of discrete measurements prior to the installation of continuously recording data loggers.

The USGS, in cooperation with the ABCWUA, the New Mexico Office of the State Engineer (NMOSE), and Bernalillo County, measures water levels from the previously defined groundwater monitoring network (table 1). Monitoring-well locations within the basin and adjacent areas are shown in figure 1, and those within the Albuquerque metropolitan area are shown in figure 2. The data presented in this report are available in the USGS National Water Information System (U.S. Geological Survey, 2020).

\section{Purpose and Scope}

The purpose of this report is to present groundwater-level data collected from the Albuquerque Basin well network during the 2019 water year (October 1, 2018, through September 30, 2019). Measurements at certain wells have been discontinued due to various reasons; groundwater-level data that were collected from those discontinued wells in previous water years can be found in previous USGS reports (Kues, 1987; Rankin, 1994, 1996, 1998, 1999, 2000; DeWees, 2001, 2002, 2003, 2006; Beman, 2007, 2008, 2009, 2011, 2012, 2013, 2014, 2015; Beman and Torres, 2010; Beman and Bryant, 2016; Beman and others, 2019; Ritchie and Galanter, 2019). A water year is the 12-month period from October 1 through September 30 and is designated by the calendar year in which it ends.

\section{Well-Numbering System}

The system of numbering wells and piezometers in New Mexico is based on the common subdivision of public lands into sections (fig. 3). Each well number, in addition to designating the well, locates the position to the nearest 10-acre tract in the public land survey system. This number (referred to as "local identifier" in table 1) is divided into four segments. The first segment denotes the township ("T.") north or south of the New Mexico base line, the second denotes the range ("R.") east or west of the New Mexico principal meridian, and the third denotes the section. The fourth segment of the well number, which consists of three digits, denotes the 160-, $40-$, and 10-acre tracts in which the well is located. Each section is divided into quarters - numbered 1, 2, 3, and 4-for the northwest, northeast, southwest, and southeast quarters, respectively. The first digit of the fourth segment gives the quarter section, which is a tract of 160 acres. Each quarter section is then subdivided into four 40-acre tracts numbered in the same manner, and the second digit denotes the 40-acre tract. Finally, each 40 -acre tract is further subdivided into four 10 -acre tracts, and the third digit denotes the 10 -acre tract. The fourth segment of the well number can further denote subdivisions of the 10-acre tract by including more than three digits; each additional digit further subdivides the tract by quarters in the same manner as shown in figure 3. Letters A, B, $\mathrm{C}$, and so on are added to the end of the last segment of the well number to designate the second, third, fourth, and succeeding wells in the same tract. For example, well 09N.03E.07.131A is the first subsequent well in the northwest quarter (NW 1/4) of the southwest quarter (SW 1/4) of the northwest quarter (NW 1/4) of section 7, T. 09 N., R. 03 E. (Bureau of Land Management, 2009).

\section{Methods}

Water-level measurements at the groundwater monitoring network were collected during the 2019 water year by following standard USGS protocols for discrete and continuous water-level measurements using electric and steel tapes and continuously recording data loggers (Cunningham and Schalk, 2011). Discrete measurements are collected at 54 of the sites (table 1); 49 sites $(2,4,9,10,12-20,24-30,32-41,46-51$, $53-55,68,70-73,76,81,83,87$, and 237) are monitored semiannually, two sites (230 and 231) are monitored quarterly, and three sites (222-224) are monitored every 2 to 3 months. At 66 of the sites $(42-45,64,165-184,189-194,196-203$, 205-221, 225-229, and 232-236; table 1), pressure transducers and data loggers are used to collect continuous (hourly) waterlevel data.

\section{Water-Level Data}

Data for the groundwater monitoring network listed in table 1 include site number, number of the figure on which the well location is shown, USGS site number, local identifier, other identifier (if applicable), latitude and longitude, well depth, screened interval, and method of water-level measurement that is shown in figure 4. Hydrographs presenting water-level data collected by the USGS include water level in feet below land surface and water level in feet above the North American Vertical Datum of 1988 (NAVD 88) (fig. 4). Data in hydrographs from wells that have continuous (hourly) 
Table 1. Data for selected wells and piezometers in and adjacent to the Albuquerque Basin, central New Mexico.

[USGS, U.S. Geological Survey; --, no data or not applicable; C, continuous measurement; D, discrete measurement. Latitude and longitude are in decimal degrees and in conformance with the North American Datum of 1983. Discontinuity in numbering sequence is due to wells omitted from this report because of lack of recent data collection. Data from discontinued wells can be seen in previous USGS open-file reports and data series (Kues, 1987; Rankin, 1994, 1996, 1998, 1999, 2000; DeWees, 2001, 2002, 2003, 2006; Beman, 2007, 2008, 2009, 2011, 2012, 2013, 2014, 2015; Beman and Torres, 2010; Beman and Bryant, 2016; Beman and others, 2019)]

\begin{tabular}{|c|c|c|c|c|c|c|c|c|c|}
\hline $\begin{array}{c}\text { Site } \\
\text { number }\end{array}$ & $\begin{array}{l}\text { Figure } \\
\text { number }\end{array}$ & $\begin{array}{c}\text { USGS } \\
\text { site number }\end{array}$ & $\begin{array}{l}\text { Local } \\
\text { identifier }\end{array}$ & $\begin{array}{c}\text { Other } \\
\text { identifier }\end{array}$ & Latitude & Longitude & $\begin{array}{l}\text { Well depth } \\
\text { (feet below } \\
\text { land surface) }\end{array}$ & $\begin{array}{c}\text { Screened } \\
\text { interval } \\
\text { (feet below } \\
\text { land surface) }\end{array}$ & $\begin{array}{c}\text { Method of } \\
\text { water-level } \\
\text { measurement } \\
\text { shown in figure } 4\end{array}$ \\
\hline 2 & 1 & 341528106533301 & 01S.01W.01.213 & -- & 34.25784459 & -106.89308333 & 38 & -- & $\mathrm{D}$ \\
\hline 4 & 1 & 342107106530401 & 02N.01E.31.313 & $\begin{array}{c}\text { Sevilleta Refuge } \\
\text { Headquarters }\end{array}$ & 34.35200976 & -106.88502789 & 223 & $210-220$ & $\mathrm{D}$ \\
\hline 9 & 1 & 344258106460901 & 06N.02E.30.412A & Estes 1 & 34.71616861 & -106.76974946 & 135 & $125-130$ & $\mathrm{D}$ \\
\hline 10 & 1 & 344258106460902 & 06N.02E.30.412B & Estes 5 & 34.71616861 & -106.76974946 & 300 & $265-270$ & $\mathrm{D}$ \\
\hline 12 & 2 & 350137106410501 & 09N.02E.12.214A & Rio Bravo Nest 1 & 35.02699256 & -106.68530346 & 149 & $139-144$ & $\mathrm{D}$ \\
\hline 13 & 2 & 350137106410502 & 09N.02E.12.214B & Rio Bravo Nest 1 & 35.02699256 & -106.68530346 & 104 & 94-99 & $\mathrm{D}$ \\
\hline 14 & 2 & 350137106410503 & 09N.02E.12.214C & Rio Bravo Nest 1 & 35.02699256 & -106.68530346 & 38 & $28-33$ & $\mathrm{D}$ \\
\hline 15 & 2 & 350138106395501 & 09N.03E.07.131A & Rio Bravo Nest 2 & 35.02727042 & -106.66585862 & 154 & $144-149$ & $\mathrm{D}$ \\
\hline 16 & 2 & 350138106395502 & 09N.03E.07.131B & Rio Bravo Nest 2 & 35.02727042 & -106.66585862 & 91 & $81-86$ & $\mathrm{D}$ \\
\hline 17 & 2 & 350138106395503 & 09N.03E.07.131C & Rio Bravo Nest 2 & 35.02727042 & -106.66585862 & 49 & $39-44$ & $\mathrm{D}$ \\
\hline 18 & 2 & 350138106393201 & 09N.03E.07.241A & Rio Bravo Nest 3 & 35.02727045 & -106.65946960 & 148 & $138-143$ & $\mathrm{D}$ \\
\hline 19 & 2 & 350138106393202 & 09N.03E.07.241B & Rio Bravo Nest 3 & 35.02727045 & -106.65946960 & 101 & 91-96 & $\mathrm{D}$ \\
\hline 20 & 2 & 350138106393203 & 09N.03E.07.241C & Rio Bravo Nest 3 & 35.02727045 & -106.65946960 & 49 & $39-44$ & $\mathrm{D}$ \\
\hline 24 & 2 & 350138106401103 & 09N.03E.07.114B & Rio Bravo Nest 5 & 35.02780278 & -106.67106944 & 515 & $500-510$ & $\mathrm{D}$ \\
\hline 25 & 2 & 350138106401101 & 09N.03E.07.114 & Rio Bravo Nest 5 & 35.02781111 & -106.67103333 & 150 & $135-145$ & $\mathrm{D}$ \\
\hline 26 & 2 & 350138106401102 & 09N.03E.07.114A & Rio Bravo Nest 5 & 35.02778611 & -106.67103611 & 22 & $7-17$ & $\mathrm{D}$ \\
\hline 27 & 2 & 350854106403701 & $11 \mathrm{~N} .02 \mathrm{E} .25 .341 \mathrm{~A}$ & Montaño Nest 1 & 35.14837767 & -106.67752663 & 152 & $140-145$ & $\mathrm{D}$ \\
\hline 28 & 2 & 350854106403702 & 11N.02E.25.341B & Montaño Nest 1 & 35.14837767 & -106.67752663 & 93 & 83-88 & $\mathrm{D}$ \\
\hline 29 & 2 & 350854106403703 & 11N.02E.25.341C & Montaño Nest 1 & 35.14837767 & -106.67752663 & 48 & $40-45$ & $\mathrm{D}$ \\
\hline 30 & 2 & 350836106395601 & 11N.03E.31.122A & Montaño Nest 2 & 35.14337788 & -106.66613746 & 147 & $138-143$ & $\mathrm{D}$ \\
\hline 32 & 2 & 350836106395603 & 11N.03E.31.122C & Montaño Nest 2 & 35.14337788 & -106.66613746 & 40 & $30-35$ & $\mathrm{D}$ \\
\hline 33 & 2 & 350827106391301 & 11N.03E.32.132A & Montaño Nest 3 & 35.14087802 & -106.65419275 & 150 & $140-145$ & $\mathrm{D}$ \\
\hline 34 & 2 & 350827106391302 & 11N.03E.32.132B & Montaño Nest 3 & 35.14087802 & -106.65419275 & 99 & $90-95$ & $\mathrm{D}$ \\
\hline 35 & 2 & 350827106391303 & 11N.03E.32.132C & Montaño Nest 3 & 35.14087802 & -106.65419275 & 50 & $40-45$ & $\mathrm{D}$ \\
\hline 36 & 2 & 350821106383701 & 11N.03E.32.234A & Montaño Nest 4 & 35.13921145 & -106.64419253 & 132 & $123-128$ & $\mathrm{D}$ \\
\hline 37 & 2 & 350821106383702 & 11N.03E.32.234B & Montaño Nest 4 & 35.13921145 & -106.64419253 & 94 & $85-90$ & $\mathrm{D}$ \\
\hline 38 & 2 & 350821106383703 & 11N.03E.32.234C & Montaño Nest 4 & 35.13937811 & -106.64455365 & 50 & $40-45$ & $\mathrm{D}$ \\
\hline
\end{tabular}


Table 1. Data for selected wells and piezometers in and adjacent to the Albuquerque Basin, central New Mexico.-Continued

[USGS, U.S. Geological Survey; --, no data or not applicable; C, continuous measurement; D, discrete measurement. Latitude and longitude are in decimal degrees and in conformance with the North American Datum of 1983. Discontinuity in numbering sequence is due to wells omitted from this report because of lack of recent data collection. Data from discontinued wells can be seen in previous USGS open-file reports and data series (Kues, 1987; Rankin, 1994, 1996, 1998, 1999, 2000; DeWees, 2001, 2002, 2003, 2006; Beman, 2007, 2008, 2009, 2011, 2012, 2013, 2014, 2015; Beman and Torres, 2010; Beman and Bryant, 2016; Beman and others, 2019)]

\begin{tabular}{|c|c|c|c|c|c|c|c|c|c|}
\hline $\begin{array}{c}\text { Site } \\
\text { number }\end{array}$ & $\begin{array}{l}\text { Figure } \\
\text { number }\end{array}$ & $\begin{array}{c}\text { USGS } \\
\text { site number }\end{array}$ & $\begin{array}{l}\text { Local } \\
\text { identifier }\end{array}$ & $\begin{array}{c}\text { Other } \\
\text { identifier }\end{array}$ & Latitude & Longitude & $\begin{array}{l}\text { Well depth } \\
\text { (feet below } \\
\text { land surface) }\end{array}$ & $\begin{array}{c}\text { Screened } \\
\text { interval } \\
\text { (feet below } \\
\text { land surface) }\end{array}$ & $\begin{array}{c}\text { Method of } \\
\text { water-level } \\
\text { measurement } \\
\text { shown in figure } 4\end{array}$ \\
\hline 39 & 2 & 350859106401601 & 11N.03E.30.313 & Montaño Nest 5 & 35.14976654 & -106.67169319 & 25 & $10-20$ & $\mathrm{D}$ \\
\hline 40 & 2 & 350859106401602 & 11N.03E.30.313A & Montaño Nest 5 & 35.14976654 & -106.67169319 & 75 & $60-70$ & $\mathrm{D}$ \\
\hline 41 & 2 & 350859106401603 & 11N.03E.30.313B & Montaño Nest 5 & 35.14976654 & -106.67169319 & 150 & $135-145$ & $\mathrm{D}$ \\
\hline 42 & 2 & 350836106395401 & 11N.03E.31.21311A & Montaño Nest 6 & 35.14298611 & -106.66544444 & 983 & $972-978$ & $\mathrm{C}$ and $\mathrm{D}$ \\
\hline 43 & 2 & 350836106395402 & 11N.03E.31.21311B & Montaño Nest 6 & 35.14298611 & -106.66544444 & 836 & $826-831$ & $\mathrm{C}$ and $\mathrm{D}$ \\
\hline 44 & 2 & 350836106395403 & 11N.03E.31.21311C & Montaño Nest 6 & 35.14298611 & -106.66544444 & 568 & $558-563$ & $\mathrm{C}$ and $\mathrm{D}$ \\
\hline 45 & 2 & 350836106395404 & 11N.03E.31.21311D & Montaño Nest 6 & 35.14298611 & -106.66544444 & 182 & $172-177$ & $\mathrm{C}$ and $\mathrm{D}$ \\
\hline 46 & 2 & 351059106385903 & 11N.03E.17.141B & Paseo del Norte Nest 1 & 35.18289722 & -106.65065556 & 600 & $545-555$ & D \\
\hline 47 & 2 & 351059106385901 & 11N.03E.17.141 & Paseo del Norte Nest 1 & 35.18289722 & -106.65065556 & 150 & $135-145$ & $\mathrm{D}$ \\
\hline 48 & 2 & 351059106385902 & 11N.03E.17.141A & Paseo del Norte Nest 1 & 35.18289722 & -106.65065556 & 25 & $10-20$ & $\mathrm{D}$ \\
\hline 49 & 2 & 351057106384201 & 11N.03E.17.233 & Paseo del Norte Nest 2 & 35.18254342 & -106.64558184 & 150 & $135-145$ & $\mathrm{D}$ \\
\hline 50 & 2 & 351057106384202 & 11N.03E.17.233A & Paseo del Norte Nest 2 & 35.18254342 & -106.64558184 & 95 & $80-90$ & $\mathrm{D}$ \\
\hline 51 & 2 & 351057106384203 & 11N.03E.17.233B & Paseo del Norte Nest 2 & 35.18254342 & -106.64558184 & 45 & $30-40$ & $\mathrm{D}$ \\
\hline 53 & 2 & 351035106364703 & 11N.03E.15.344C & Paseo del Norte Nest 3 & 35.17686944 & -106.61366667 & 544 & $539-544$ & $\mathrm{D}$ \\
\hline 54 & 2 & 351035106364702 & 11N.03E.15.344B & Paseo del Norte Nest 3 & 35.17686944 & -106.61366667 & 144 & $139-144$ & $\mathrm{D}$ \\
\hline 55 & 2 & 351035106364701 & 11N.03E.15.344A & Paseo del Norte Nest 3 & 35.17686944 & -106.61366667 & 69 & $64-69$ & $\mathrm{D}$ \\
\hline 64 & 2 & 350256106390801 & 10N.03E.32.314 & San Jose 9 & 35.04897222 & -106.65331944 & 765 & $189-765$ & $\mathrm{C}$ \\
\hline 68 & 1 & 350602106210401 & 10N.05E.12.434 & Home Oil & 35.10060157 & -106.35169117 & 54 & -- & $\mathrm{D}$ \\
\hline 70 & 2 & 350548106383901 & 10N.03E.17.232 & City 1 & 35.09643056 & -106.64495000 & 149 & $139-149$ & $\mathrm{D}$ \\
\hline 71 & 2 & 350824106375301 & 11N.03E.33.143 & City 2 & 35.14004482 & -106.63197006 & 150 & $140-150$ & $\mathrm{D}$ \\
\hline 72 & 2 & 350837106393801 & 11 N.03E.31.214 & City 3 & 35.14337791 & -106.66113736 & 152 & $142-152$ & $\mathrm{D}$ \\
\hline 73 & 2 & 350646106403601 & 10N.02E.12.241 & City 4 & 35.11282323 & -106.67724852 & 150 & $140-150$ & $\mathrm{D}$ \\
\hline 76 & 1 & 350454106570401 & 10N.01W.21.134 & Cañoncito & 35.08171142 & -106.95170006 & 117 & -- & $\mathrm{D}$ \\
\hline 81 & 1 & 351852106344901 & 13N.03E.36.132A & San Miguel & 35.31473333 & -106.58128611 & 206 & -- & $\mathrm{D}$ \\
\hline 83 & 2 & 350829106420401 & 11N.02E.35.142 & La Luz del Sol & 35.14341389 & -106.70094722 & 250 & $230-245$ & $\mathrm{D}$ \\
\hline 87 & 2 & 351009106344701 & 11N.03E.24.142 & Pino Yards & 35.16921082 & -106.58030259 & 360 & $320-360$ & $\mathrm{D}$ \\
\hline 165 & 2 & 350908106344401 & 11N.03E.25.322 & Sister Cities & 35.15256667 & -106.57949167 & 1,308 & $1,298-1,303$ & $\mathrm{C}$ \\
\hline 166 & 2 & 350908106344402 & $11 \mathrm{~N} .03 \mathrm{E} .25 .322 \mathrm{~A}$ & Sister Cities & 35.15256667 & -106.57949167 & 799 & 789-794 & $\mathrm{C}$ \\
\hline
\end{tabular}


Table 1. Data for selected wells and piezometers in and adjacent to the Albuquerque Basin, central New Mexico.-Continued

[USGS, U.S. Geological Survey; --, no data or not applicable; C, continuous measurement; D, discrete measurement. Latitude and longitude are in decimal degrees and in conformance with the North American Datum of 1983. Discontinuity in numbering sequence is due to wells omitted from this report because of lack of recent data collection. Data from discontinued wells can be seen in previous USGS open-file reports and data series (Kues, 1987; Rankin, 1994, 1996, 1998, 1999, 2000; DeWees, 2001, 2002, 2003, 2006; Beman, 2007, 2008, 2009, 2011, 2012, 2013, 2014, 2015; Beman and Torres, 2010; Beman and Bryant, 2016; Beman and others, 2019)]

\begin{tabular}{|c|c|c|c|c|c|c|c|c|c|}
\hline $\begin{array}{c}\text { Site } \\
\text { number }\end{array}$ & $\begin{array}{l}\text { Figure } \\
\text { number }\end{array}$ & $\begin{array}{c}\text { USGS } \\
\text { site number }\end{array}$ & $\begin{array}{c}\text { Local } \\
\text { identifier }\end{array}$ & $\begin{array}{c}\text { Other } \\
\text { identifier }\end{array}$ & Latitude & Longitude & $\begin{array}{l}\text { Well depth } \\
\text { (feet below } \\
\text { land surface) }\end{array}$ & $\begin{array}{c}\text { Screened } \\
\text { interval } \\
\text { (feet below } \\
\text { land surface) }\end{array}$ & $\begin{array}{c}\text { Method of } \\
\text { water-level } \\
\text { measurement } \\
\text { shown in figure } 4\end{array}$ \\
\hline 167 & 2 & 350534106354701 & 10N.03E.14.324 & Del Sol Divider & 35.09302222 & -106.59702778 & 1,567 & $1,557-1,562$ & $\mathrm{C}$ \\
\hline 168 & 2 & 350534106354702 & 10N.03E.14.324A & Del Sol Divider & 35.09302222 & -106.59702778 & 842 & $832-837$ & $\mathrm{C}$ \\
\hline 169 & 2 & 350534106354703 & 10N.03E.14.324B & Del Sol Divider & 35.09302222 & -106.59702778 & 425 & $315-415$ & $\mathrm{C}$ \\
\hline 170 & 1 & 351201106400501 & 11N.03E.07.141 & Hunters Ridge Nest 1 & 35.20005278 & -106.66900556 & 1,518 & $1,508-1,513$ & $\mathrm{C}$ \\
\hline 171 & 1 & 351201106400502 & 11N.03E.07.141A & Hunters Ridge Nest 1 & 35.20005278 & -106.66900556 & 855 & $845-850$ & $\mathrm{C}$ \\
\hline 172 & 1 & 351201106400503 & 11N.03E.07.141B & Hunters Ridge Nest 1 & 35.20005278 & -106.66900556 & 238 & $148-228$ & $\mathrm{C}$ \\
\hline 173 & 1 & 351201106400504 & 11N.03E.07.141C & Hunters Ridge Nest 2 & 35.20005278 & -106.66900556 & 359 & $349-354$ & $\mathrm{C}$ \\
\hline 174 & 1 & 351201106400505 & $11 \mathrm{~N} .03 \mathrm{E} .07 .141 \mathrm{D}$ & Hunters Ridge Nest 2 & 35.20005278 & -106.66900556 & 305 & $295-300$ & $\mathrm{C}$ \\
\hline 175 & 1 & 351201106400506 & 11N.03E.07.141E & Hunters Ridge Nest 2 & 35.20005278 & -106.66900556 & 263 & $238-258$ & $\mathrm{C}$ \\
\hline 176 & 2 & 350638106413701 & 10N.02E.11.244 & West Bluff Nest 1 & 35.11000556 & -106.69462222 & 1,095 & $1,085-1,090$ & $\mathrm{C}$ \\
\hline 177 & 2 & 350638106413702 & 10N.02E.11.244A & West Bluff Nest 1 & 35.11000556 & -106.69462222 & 689 & 679-684 & $\mathrm{C}$ \\
\hline 178 & 2 & 350638106413703 & 10N.02E.11.244B & West Bluff Nest 1 & 35.11000556 & -106.69462222 & 433 & $422-427$ & $\mathrm{C}$ \\
\hline 179 & 2 & 350638106413704 & 10N.02E.11.244C & West Bluff Nest 2 & 35.11000556 & -106.69462222 & 328 & $318-323$ & $\mathrm{C}$ \\
\hline 180 & 2 & 350638106413705 & 10N.02E.11.244D & West Bluff Nest 2 & 35.11000556 & -106.69462222 & 254 & $244-249$ & $\mathrm{C}$ \\
\hline 181 & 2 & 350638106413706 & 10N.02E.11.244E & West Bluff Nest 2 & 35.11000556 & -106.69462222 & 173 & $143-163$ & $\mathrm{C}$ \\
\hline 182 & 2 & 350706106390301 & 10N.03E.05.341 & Garfield Park & 35.11798611 & -106.65127222 & 1,020 & $995-1,010$ & $\mathrm{C}$ \\
\hline 183 & 2 & 350706106390302 & 10N.03E.05.341A & Garfield Park & 35.11798611 & -106.65127222 & 582 & $552-572$ & $\mathrm{C}$ \\
\hline 184 & 2 & 350706106390303 & 10N.03E.05.341B & Garfield Park & 35.11798611 & -106.65127222 & 93 & $43-83$ & $\mathrm{C}$ \\
\hline 189 & 2 & 351114106330601 & 11N.04E.18.222 & Nor Este & 35.18660833 & -106.55321111 & 1,525 & $1,515-1,520$ & $\mathrm{C}$ \\
\hline 190 & 2 & 351114106330602 & $11 \mathrm{~N} .04 \mathrm{E} .18 .222 \mathrm{~A}$ & Nor Este & 35.18660833 & -106.55321111 & 1,193 & $1,183-1,188$ & $\mathrm{C}$ \\
\hline 191 & 2 & 351114106330603 & 11N.04E.18.222B & Nor Este & 35.18660833 & -106.55321111 & 608 & $538-598$ & $\mathrm{C}$ \\
\hline 192 & 2 & 350910106414801 & 11N.03E.26.243 & Sierra Vista & 35.15273889 & -106.69651944 & 1,644 & $1,634-1,639$ & $\mathrm{C}$ \\
\hline 193 & 2 & 350910106414802 & 11N.03E.26.243A & Sierra Vista & 35.15273889 & -106.69651944 & 928 & 918-923 & $\mathrm{C}$ \\
\hline 194 & 2 & 350910106414803 & 11 N.03E.26.243B & Sierra Vista & 35.15273889 & -106.69651944 & 210 & $140-200$ & $\mathrm{C}$ \\
\hline 196 & 2 & 350056106370102 & 09N.03E.10.334A & Montessa Park & 35.01586111 & -106.61731667 & 708 & $698-703$ & $\mathrm{C}$ \\
\hline 197 & 2 & 350056106370103 & 09N.03E.10.334B & Montessa Park & 35.01586111 & -106.61731667 & 330 & $260-320$ & $\mathrm{C}$ \\
\hline 198 & 1 & 345650106415901 & 08N.02E.02.413 & Isleta & 34.94729167 & -106.69992778 & 1,340 & $1,330-1,335$ & $\mathrm{C}$ \\
\hline 199 & 1 & 345650106415902 & 08N.02E.02.413A & Isleta & 34.94729167 & -106.69992778 & 815 & 805-810 & $\mathrm{C}$ \\
\hline 200 & 1 & 345650106415903 & 08N.02E.02.413B & Isleta & 34.94729167 & -106.69992778 & 185 & $175-180$ & $\mathrm{C}$ \\
\hline 201 & 1 & 345650106415904 & 08N.02E.02.413C & Isleta & 34.94729167 & -106.69992778 & 50 & $10-40$ & $\mathrm{C}$ \\
\hline
\end{tabular}


Table 1. Data for selected wells and piezometers in and adjacent to the Albuquerque Basin, central New Mexico.-Continued

[USGS, U.S. Geological Survey; --, no data or not applicable; C, continuous measurement; D, discrete measurement. Latitude and longitude are in decimal degrees and in conformance with the North American Datum of 1983. Discontinuity in numbering sequence is due to wells omitted from this report because of lack of recent data collection. Data from discontinued wells can be seen in previous USGS open-file reports and data series (Kues, 1987; Rankin, 1994, 1996, 1998, 1999, 2000; DeWees, 2001, 2002, 2003, 2006; Beman, 2007, 2008, 2009, 2011, 2012, 2013, 2014, 2015; Beman and Torres, 2010; Beman and Bryant, 2016; Beman and others, 2019)]

\begin{tabular}{|c|c|c|c|c|c|c|c|c|c|}
\hline $\begin{array}{c}\text { Site } \\
\text { number }\end{array}$ & $\begin{array}{l}\text { Figure } \\
\text { number }\end{array}$ & $\begin{array}{c}\text { USGS } \\
\text { site number }\end{array}$ & $\begin{array}{l}\text { Local } \\
\text { identifier }\end{array}$ & $\begin{array}{c}\text { Other } \\
\text { identifier }\end{array}$ & Latitude & Longitude & $\begin{array}{l}\text { Well depth } \\
\text { (feet below } \\
\text { land surface) }\end{array}$ & $\begin{array}{c}\text { Screened } \\
\text { interval } \\
\text { (feet below } \\
\text { land surface) }\end{array}$ & $\begin{array}{c}\text { Method of } \\
\text { water-level } \\
\text { measurement } \\
\text { shown in figure } 4\end{array}$ \\
\hline 202 & 1 & 351357106323001 & 12N.04E.29.433 & Sandia Pueblo & 35.23254235 & -106.54224682 & 1,305 & $1,295-1,300$ & $\mathrm{C}$ \\
\hline 203 & 1 & 351357106323002 & 12N.04E.29.433A & Sandia Pueblo & 35.23254235 & -106.54224682 & 1,025 & $1,015-1,020$ & $\mathrm{C}$ \\
\hline 205 & 1 & 344431106393401 & 06N.03E.18.442 & Tomé & 34.74185000 & -106.66237778 & 1,200 & $1,185-1,195$ & $\mathrm{C}$ \\
\hline 206 & 1 & 344431106393402 & 06N.03E.18.442A & Tomé & 34.74185000 & -106.66237778 & 710 & $695-705$ & $\mathrm{C}$ \\
\hline 207 & 1 & 344431106393403 & 06N.03E.18.442B & Tomé & 34.74185000 & -106.66237778 & 275 & $225-265$ & $\mathrm{C}$ \\
\hline 208 & 1 & 343753106430601 & 05N.03E.28.411 & Nancy Lopez & 34.63138333 & -106.71845556 & 1,186 & $1,166-1,176$ & $\mathrm{C}$ \\
\hline 209 & 1 & 343753106430602 & 05N.03E.28.411A & Nancy Lopez & 34.63138333 & -106.71845556 & 695 & $675-685$ & $\mathrm{C}$ \\
\hline 210 & 1 & 354056106215801 & 17N.05E.24.344 & Dome Road & 35.68141751 & -106.36669452 & 1,295 & $1,280-1,290$ & $\mathrm{C}$ \\
\hline 211 & 2 & 350100106405701 & 09N.02E.12.433 & Rio Bravo Park & 35.01659444 & -106.68274722 & 595 & $585-590$ & $\mathrm{C}$ \\
\hline 212 & 2 & 350100106405702 & 09N.02E.12.433A & Rio Bravo Park & 35.01659444 & -106.68274722 & 210 & $200-205$ & $\mathrm{C}$ \\
\hline 213 & 2 & 345758106364001 & 09N.03E.34.231 & Mesa del Sol & 34.96625833 & -106.61168611 & 1,630 & $1,580-1,620$ & $\mathrm{C}$ \\
\hline 214 & 2 & 345758106364002 & 09N.03E.34.231A & Mesa del Sol & 34.96625833 & -106.61168611 & 1,015 & $990-1,010$ & $\mathrm{C}$ \\
\hline 215 & 2 & 345758106364003 & 09N.03E.34.231B & Mesa del Sol & 34.96625833 & -106.61168611 & 525 & $420-520$ & $\mathrm{C}$ \\
\hline 216 & 2 & 350653106311601 & 10N.04E.09.214 & Matheson Park & 35.11477500 & -106.52182222 & 1,520 & $1,460-1,500$ & $\mathrm{C}$ \\
\hline 217 & 2 & 350653106311602 & 10N.04E.09.214A & Matheson Park & 35.11477500 & -106.52182222 & 1,045 & $1,020-1,040$ & $\mathrm{C}$ \\
\hline 218 & 2 & 350653106311603 & 10N.04E.09.214B & Matheson Park & 35.11477500 & -106.52182222 & 705 & $600-700$ & $\mathrm{C}$ \\
\hline 219 & 1 & 351515106410401 & 12N.02E.24.144 & Lincoln Middle School & 35.25423056 & -106.68513889 & 1,260 & $1,200-1,240$ & $\mathrm{C}$ \\
\hline 220 & 1 & 351515106410402 & 12N.02E.24.144A & Lincoln Middle School & 35.25423056 & -106.68513889 & 835 & $810-830$ & $\mathrm{C}$ \\
\hline 221 & 1 & 351515106410403 & 12N.02E.24.144B & Lincoln Middle School & 35.25423056 & -106.68513889 & 595 & $490-590$ & $\mathrm{C}$ \\
\hline 222 & 1 & 345842106443101 & 09N.02E.28.312 & Niese Road & 34.97850833 & -106.74157778 & 1,455 & $1,445-1,450$ & $\mathrm{D}$ \\
\hline 223 & 1 & 345842106443102 & 09N.02E.28.312A & Niese Road & 34.97850833 & -106.74157778 & 960 & $950-955$ & $\mathrm{D}$ \\
\hline 224 & 1 & 345842106443103 & 09N.02E.28.312B & Niese Road & 34.97850833 & -106.74157778 & 297 & $242-292$ & $\mathrm{D}$ \\
\hline 225 & 1 & 350244106450201 & 10N.02E.32.433 & Westgate Heights Park & 35.04563611 & -106.75075278 & 1,290 & $1,280-1,285$ & $\mathrm{C}$ \\
\hline 226 & 1 & 350244106450202 & 10N.02E.32.433A & Westgate Heights Park & 35.04563611 & -106.75075278 & 868 & $858-863$ & $\mathrm{C}$ \\
\hline 227 & 1 & 350244106450203 & 10N.02E.32.433B & Westgate Heights Park & 35.04563611 & -106.75075278 & 370 & $320-360$ & $\mathrm{C}$ \\
\hline 228 & 1 & 351821106333901 & 13N.04E.31.343 & Bernalillo & 35.30549722 & -106.55992500 & 1,190 & $1,175-1,185$ & $\mathrm{C}$ \\
\hline 229 & 1 & 351821106333902 & 13N.04E.31.343A & Bernalillo & 35.30549722 & -106.55992500 & 320 & $300-310$ & $\mathrm{C}$ \\
\hline
\end{tabular}


Table 1. Data for selected wells and piezometers in and adjacent to the Albuquerque Basin, central New Mexico.—Continued

[USGS, U.S. Geological Survey; --, no data or not applicable; C, continuous measurement; D, discrete measurement. Latitude and longitude are in decimal degrees and in conformance with the North American Datum of 1983. Discontinuity in numbering sequence is due to wells omitted from this report because of lack of recent data collection. Data from discontinued wells can be seen in previous USGS open-file reports and data series (Kues, 1987; Rankin, 1994, 1996, 1998, 1999, 2000; DeWees, 2001, 2002, 2003, 2006; Beman, 2007, 2008, 2009, 2011, 2012, 2013, 2014, 2015; Beman and Torres, 2010; Beman and Bryant, 2016; Beman and others, 2019)]

\begin{tabular}{|c|c|c|c|c|c|c|c|c|c|}
\hline $\begin{array}{c}\text { Site } \\
\text { number }\end{array}$ & $\begin{array}{l}\text { Figure } \\
\text { number }\end{array}$ & $\begin{array}{c}\text { USGS } \\
\text { site number }\end{array}$ & $\begin{array}{l}\text { Local } \\
\text { identifier }\end{array}$ & $\begin{array}{c}\text { Other } \\
\text { identifier }\end{array}$ & Latitude & Longitude & $\begin{array}{l}\text { Well depth } \\
\text { (feet below } \\
\text { land surface) }\end{array}$ & $\begin{array}{c}\text { Screened } \\
\text { interval } \\
\text { (feet below } \\
\text { land surface) }\end{array}$ & $\begin{array}{c}\text { Method of } \\
\text { water-level } \\
\text { measurement } \\
\text { shown in figure } 4\end{array}$ \\
\hline 230 & 1 & 352019106474801 & 13N.01E.24.313 & Phoenix Road & 35.33926111 & -106.79660278 & 1,625 & $1,600-1,620$ & $\mathrm{D}$ \\
\hline 231 & 1 & 351040106482801 & 11 N.01E.14.342 & Paradise Road & 35.17772222 & -106.80766667 & 1,735 & $1,720-1,730$ & $\mathrm{D}$ \\
\hline 232 & 2 & 350545106335901 & 10N.04E.18.133A & Jerry Cline Park & 35.09531389 & -106.56608056 & 1,455 & $1,435-1,445$ & $\mathrm{C}$ \\
\hline 233 & 2 & 350545106335902 & 10N.04E.18.133B & Jerry Cline Park & 35.09531389 & -106.56608056 & 1,050 & $1,030-1,040$ & $\mathrm{C}$ \\
\hline 234 & 2 & 350545106335903 & 10N.04E.18.133C & Jerry Cline Park & 35.09531389 & -106.56608056 & 510 & $400-500$ & $\mathrm{C}$ \\
\hline 235 & 2 & 350307106410601 & 10N.02E.36.321A & Armijo & 35.05063333 & -106.68394722 & 1,623 & $1,593-1,613$ & $\mathrm{C}$ \\
\hline 236 & 2 & 350307106410602 & 10N.02E.36.321B & Armijo & 35.05063333 & -106.68394722 & 1,025 & $995-1,015$ & $\mathrm{C}$ \\
\hline 237 & 1 & 350552106444601 & 10N.02E.17.242 & Arroyo Vista & 35.09775000 & -106.74633333 & 1,424 & $520-1,424$ & $\mathrm{D}$ \\
\hline
\end{tabular}




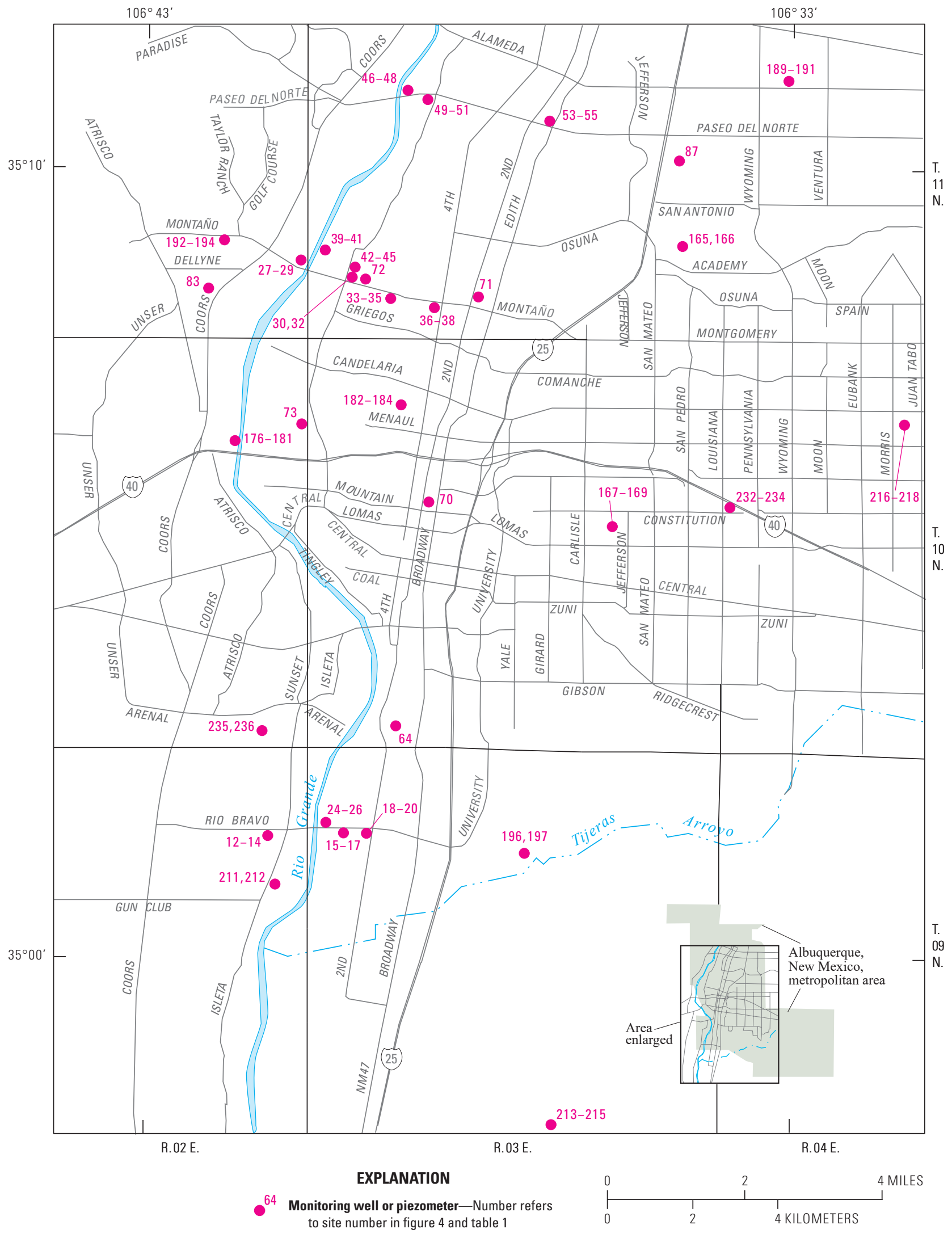

Figure 2. Location of selected monitoring wells and piezometers within the Albuquerque, New Mexico, metropolitan area. 


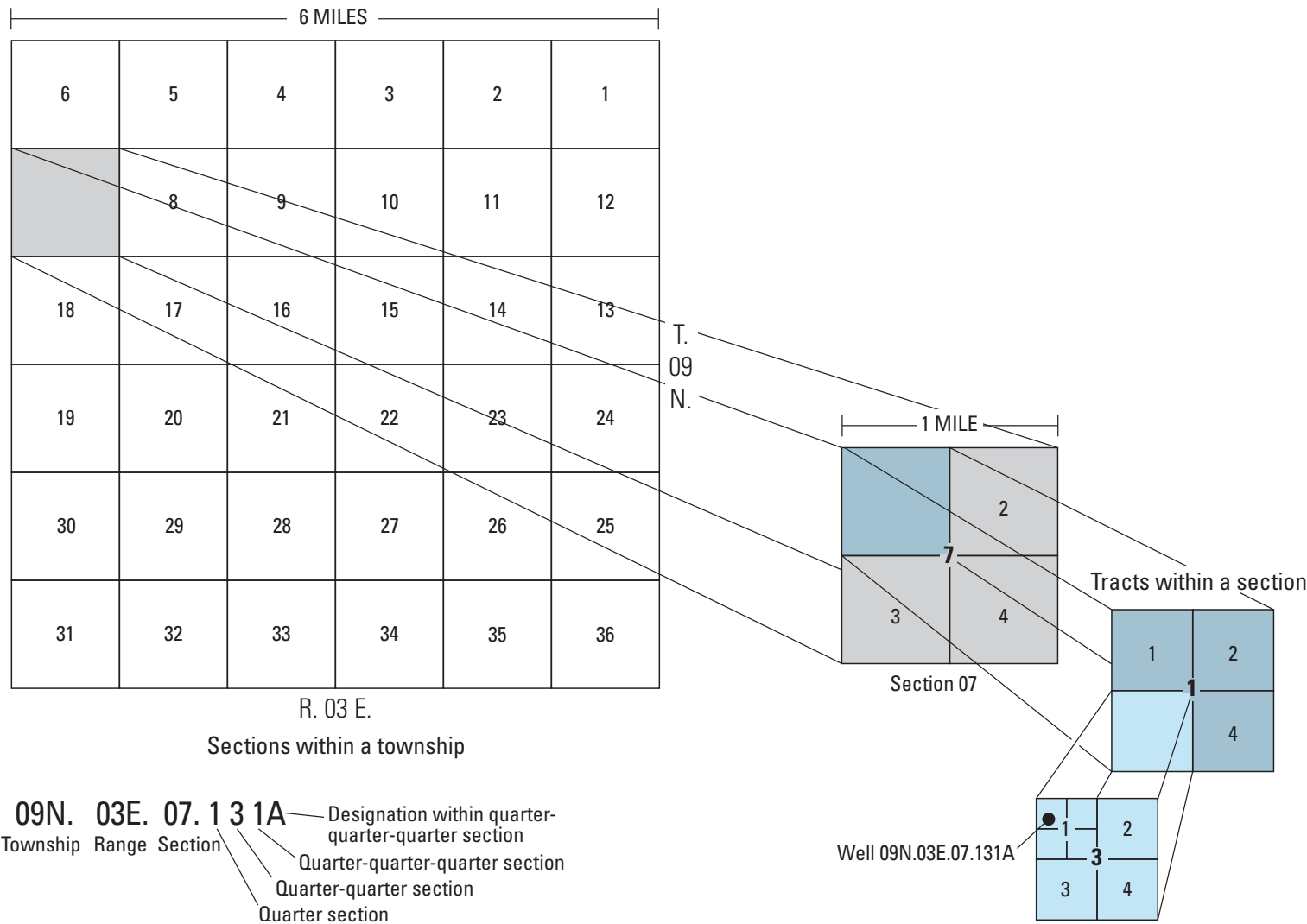

Figure 3. System for numbering wells and piezometers in New Mexico.

recorders are shown by solid lines that represent daily mean water levels (fig. 4). In hydrographs that present data from discretely measured wells, dashed lines connect symbols (“"+") that represent the discrete measurements (fig. 4). In hydrographs showing discretely measured piezometers that are nested (more than one trace per graph), the symbols were removed to make the hydrographs easier to read (fig. 4). There are two hydrographs presenting data for sites 42-45 (table 1): one showing discrete water-level measurements and one showing continuous data (fig. 4). The period of record for discrete measurements is much longer than that of continuous data in some locations. For those wells, both hydrographs have been included to ensure that all data are presented. Data gaps are present in some of the continuous hydrographs; these gaps may be caused by equipment malfunction or removal of spurious data during the review and approval process.

The transducer was removed from site 218 on July 16, 2019. There is no continuous data for that site after that date. Future versions of this report will include a graph presenting discrete measurements that will continue at that site.

The transducers and data loggers were removed from sites 222-224 in 2014, but discrete measurements have been collected at those sites since that time (table 1). Continuous data from sites 222-224 can be viewed in the previous USGS reports. 

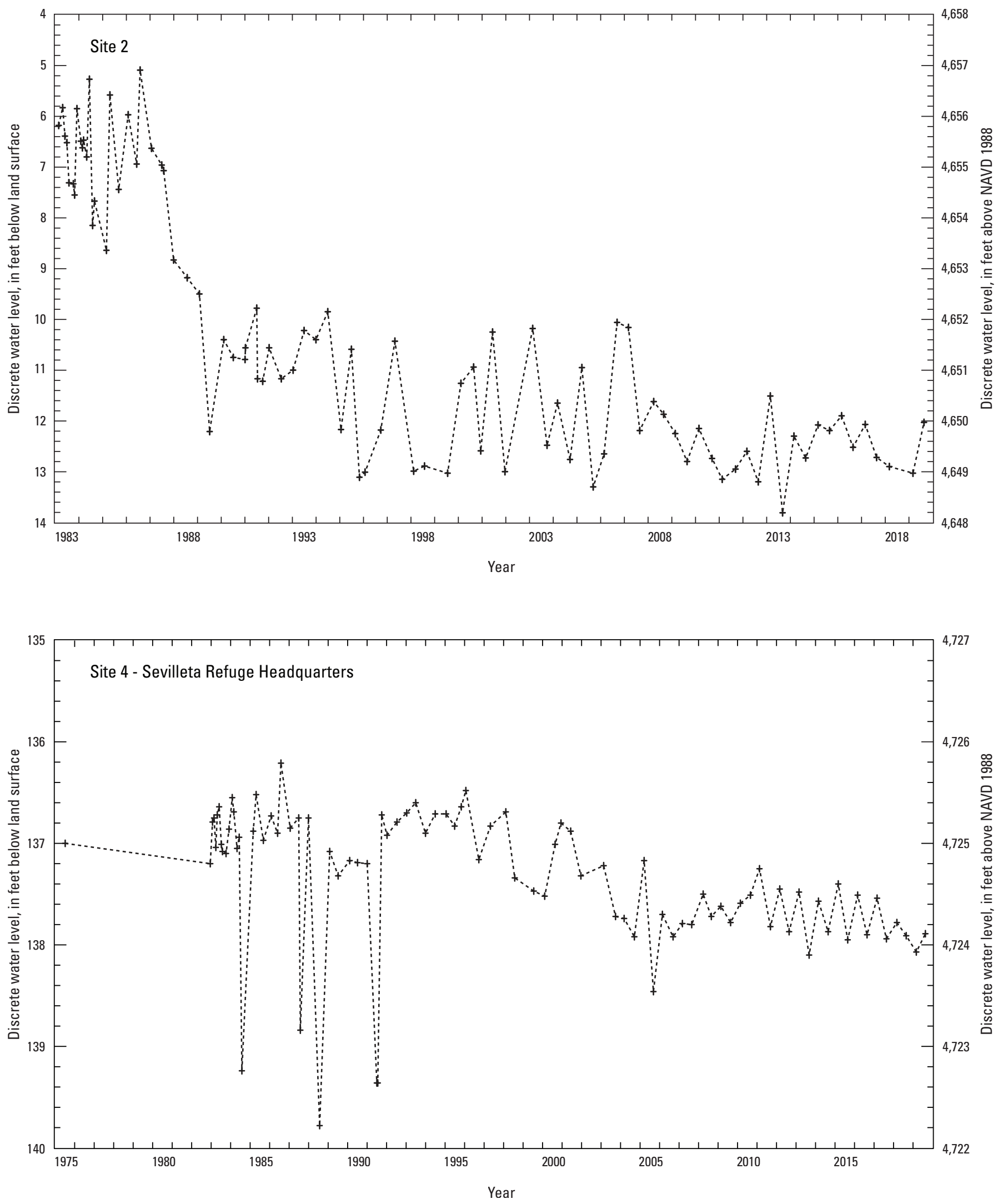

Figure 4. Water-level data for selected wells and piezometers in and adjacent to the Albuquerque Basin, central New Mexico, period of record through September 30, 2019 (NAVD 1988, North American Vertical Datum of 1988). Site numbers correspond to those in table 1. 

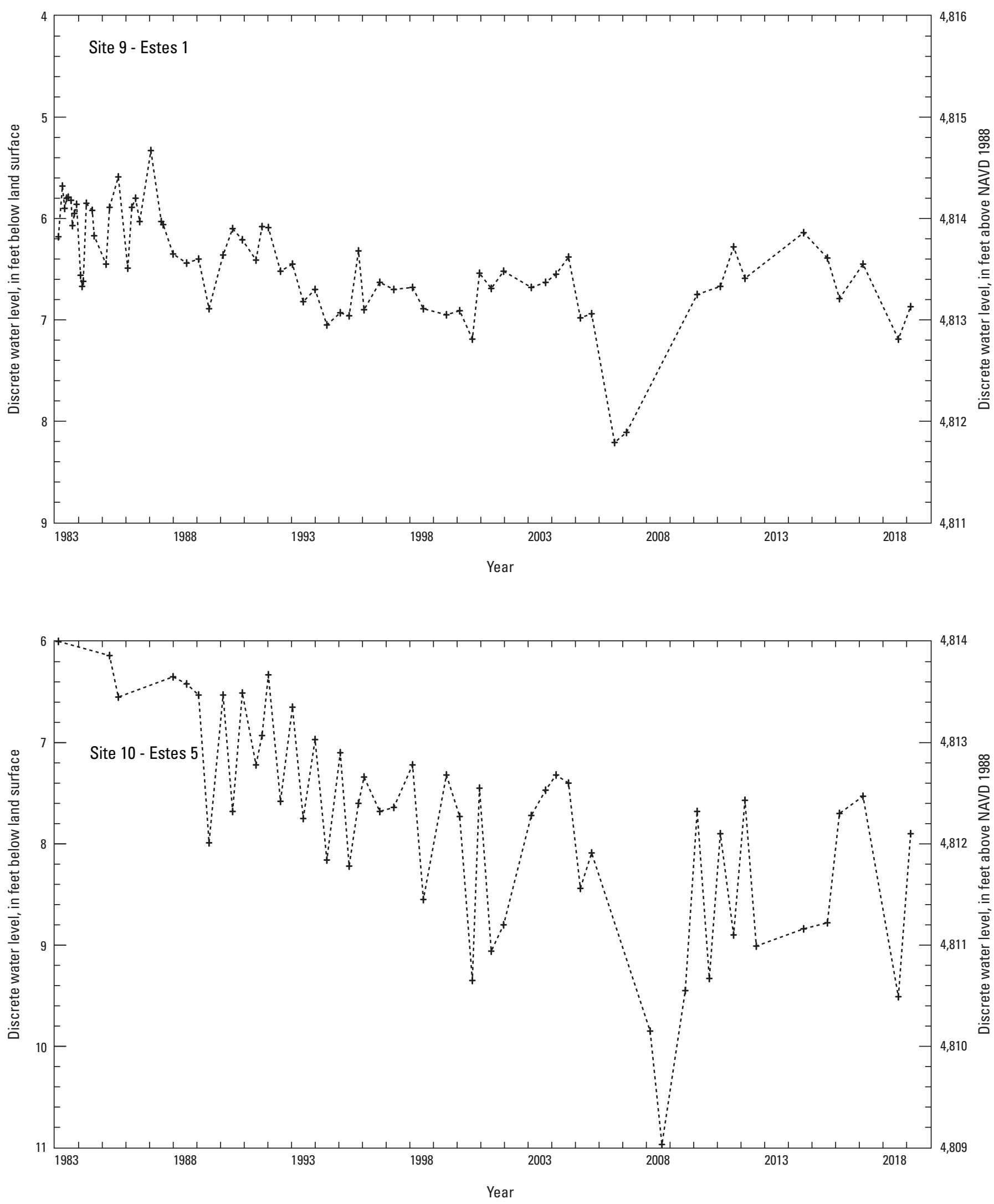

Figure 4. Water-level data for selected wells and piezometers in and adjacent to the Albuquerque Basin, central New Mexico, period of record through September 30, 2019 (NAVD 1988, North American Vertical Datum of 1988). Site numbers correspond to those in table 1.-Continued 

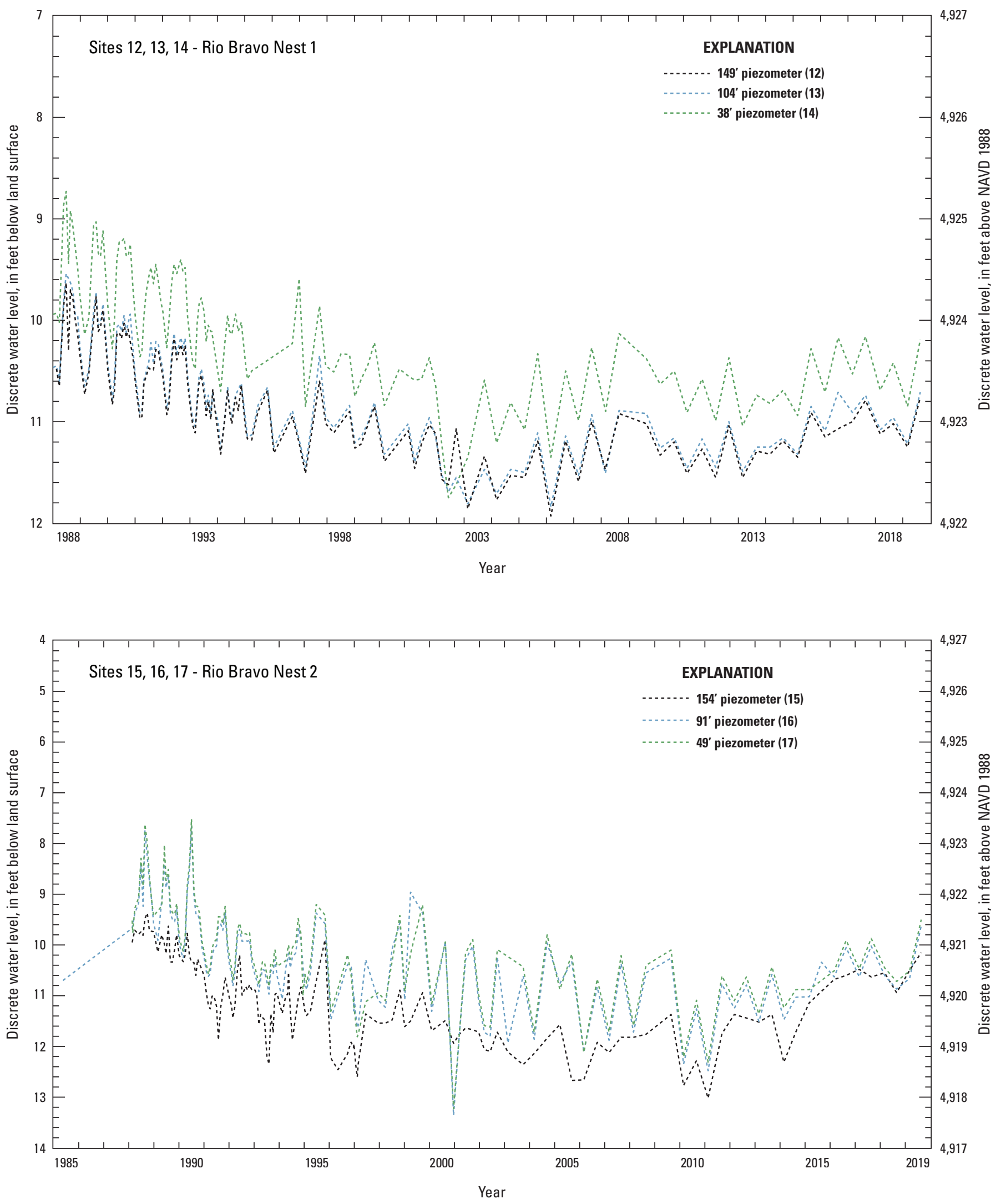

Figure 4. Water-level data for selected wells and piezometers in and adjacent to the Albuquerque Basin, central New Mexico, period of record through September 30, 2019 (NAVD 1988, North American Vertical Datum of 1988). Site numbers correspond to those in table 1.-Continued 

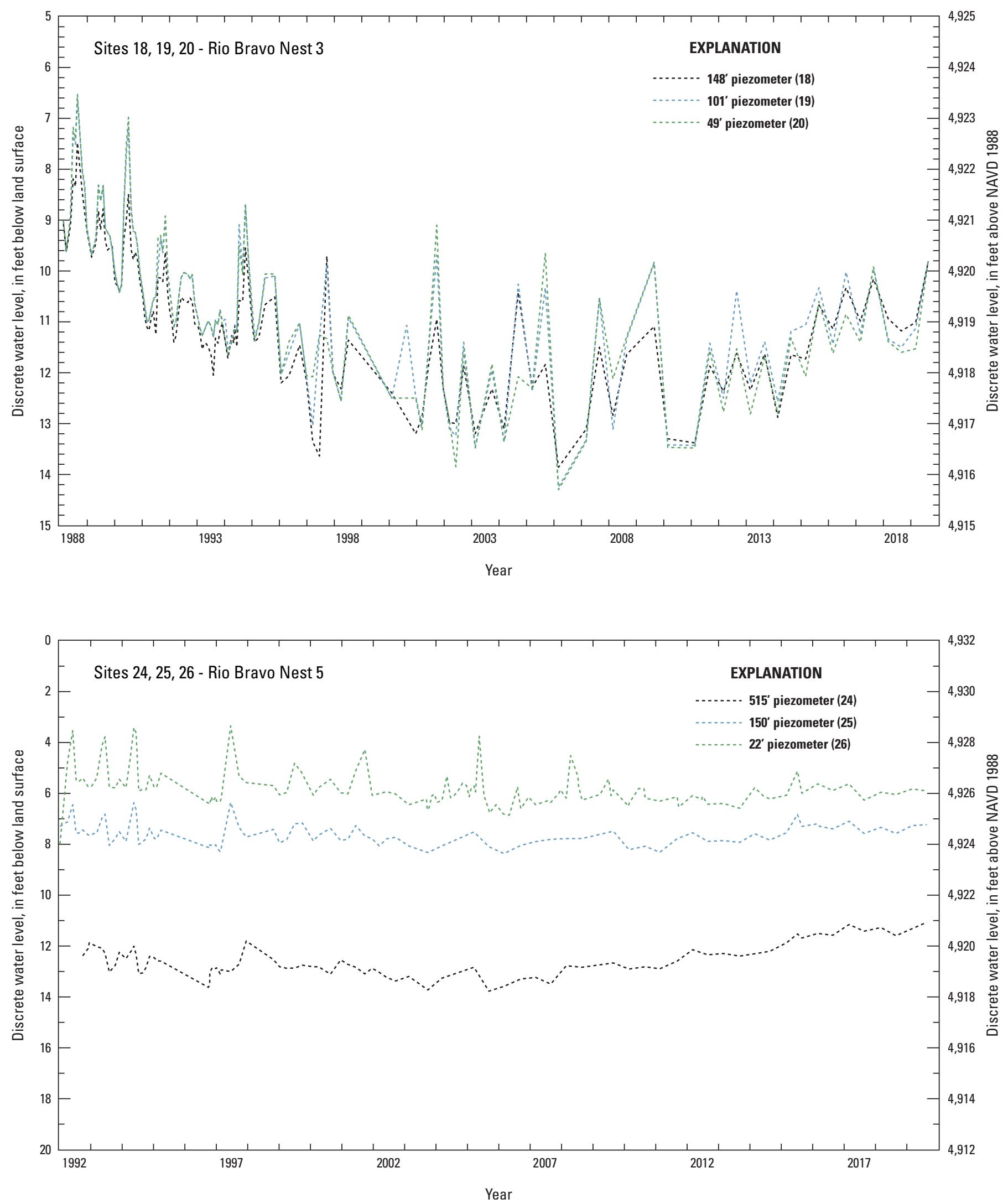

Figure 4. Water-level data for selected wells and piezometers in and adjacent to the Albuquerque Basin, central New Mexico, period of record through September 30, 2019 (NAVD 1988, North American Vertical Datum of 1988). Site numbers correspond to those in table 1.-Continued 

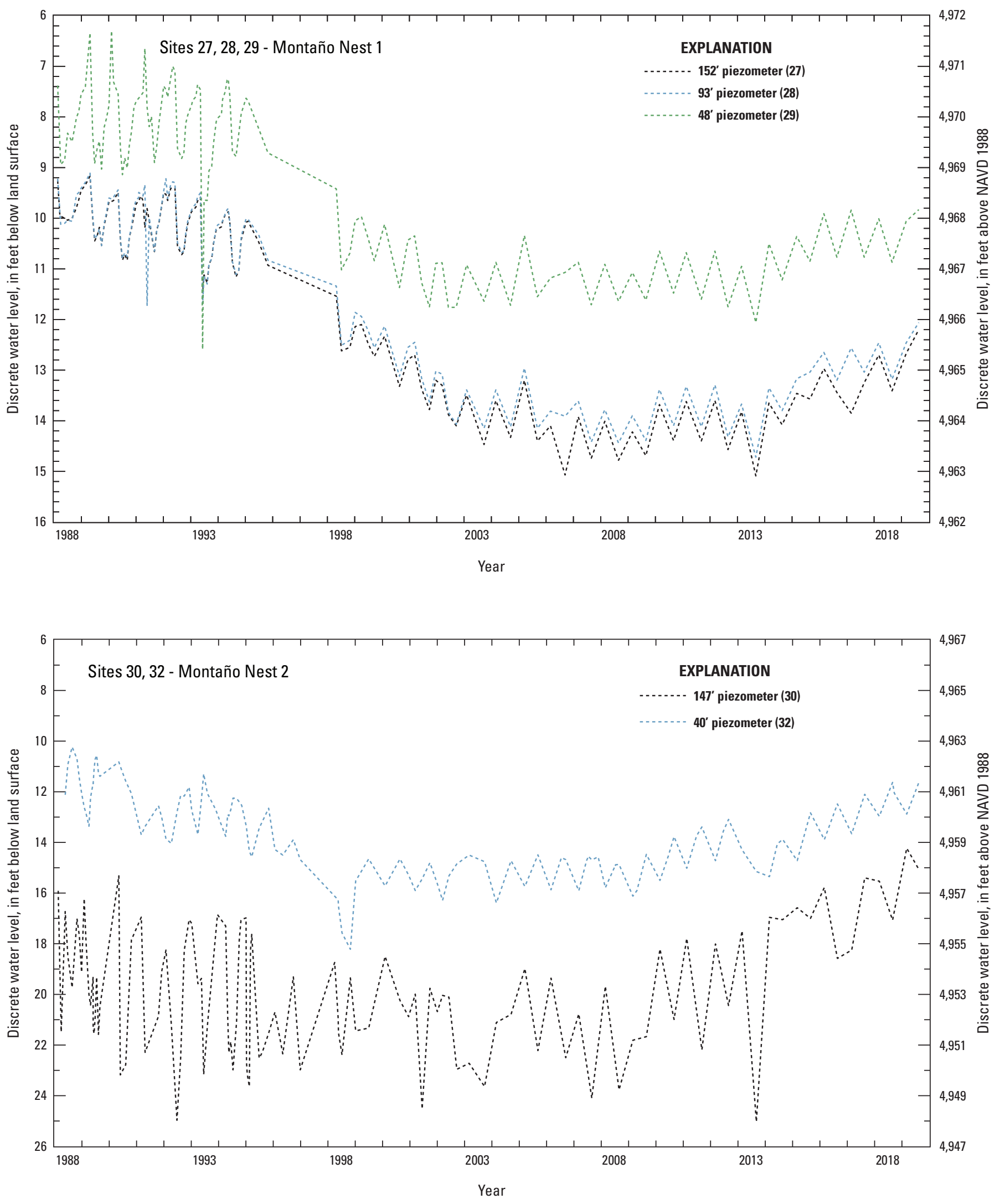

Figure 4. Water-level data for selected wells and piezometers in and adjacent to the Albuquerque Basin, central New Mexico, period of record through September 30, 2019 (NAVD 1988, North American Vertical Datum of 1988). Site numbers correspond to those in table 1.-Continued 

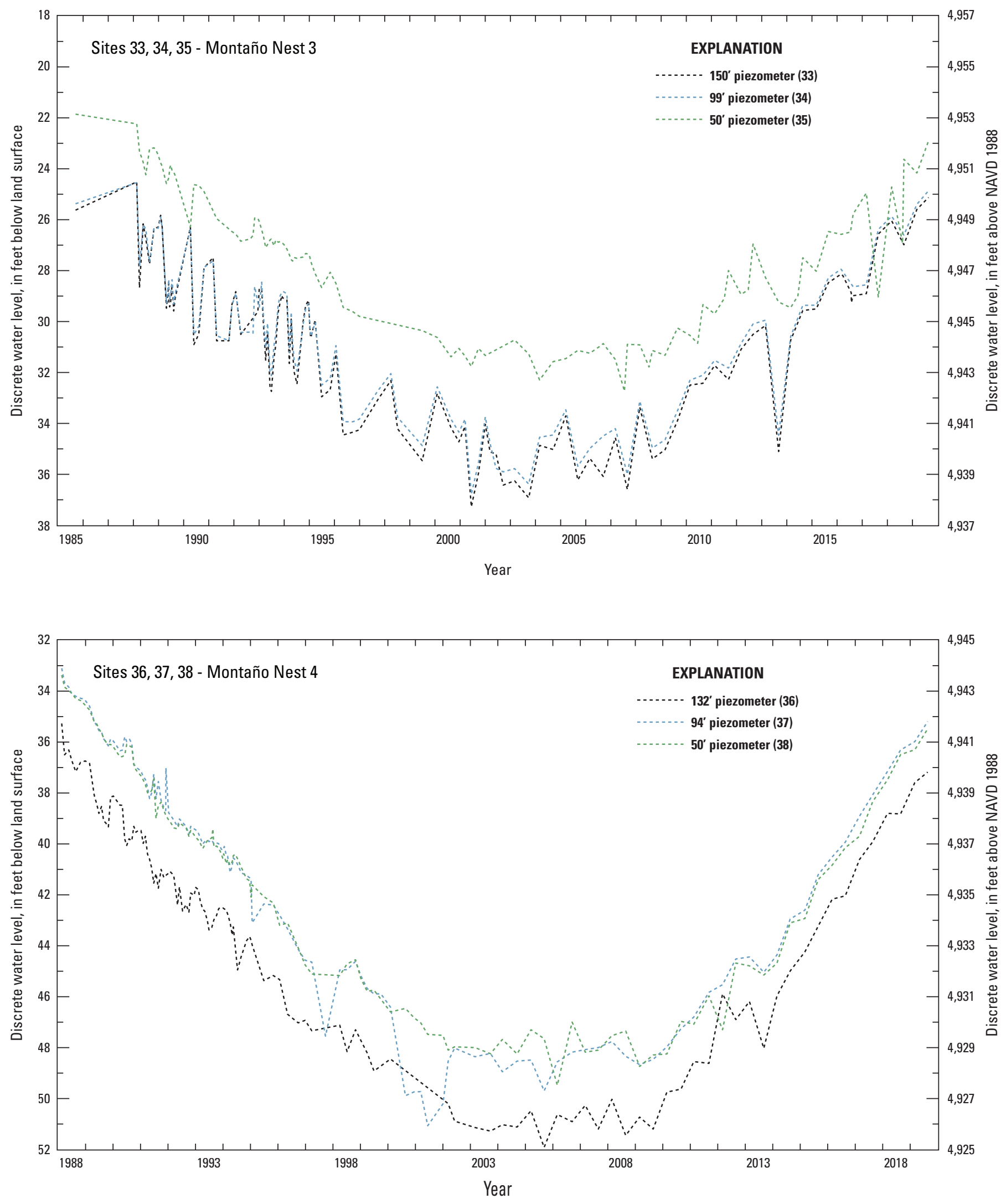

Figure 4. Water-level data for selected wells and piezometers in and adjacent to the Albuquerque Basin, central New Mexico, period of record through September 30, 2019 (NAVD 1988, North American Vertical Datum of 1988). Site numbers correspond to those in table 1.-Continued 

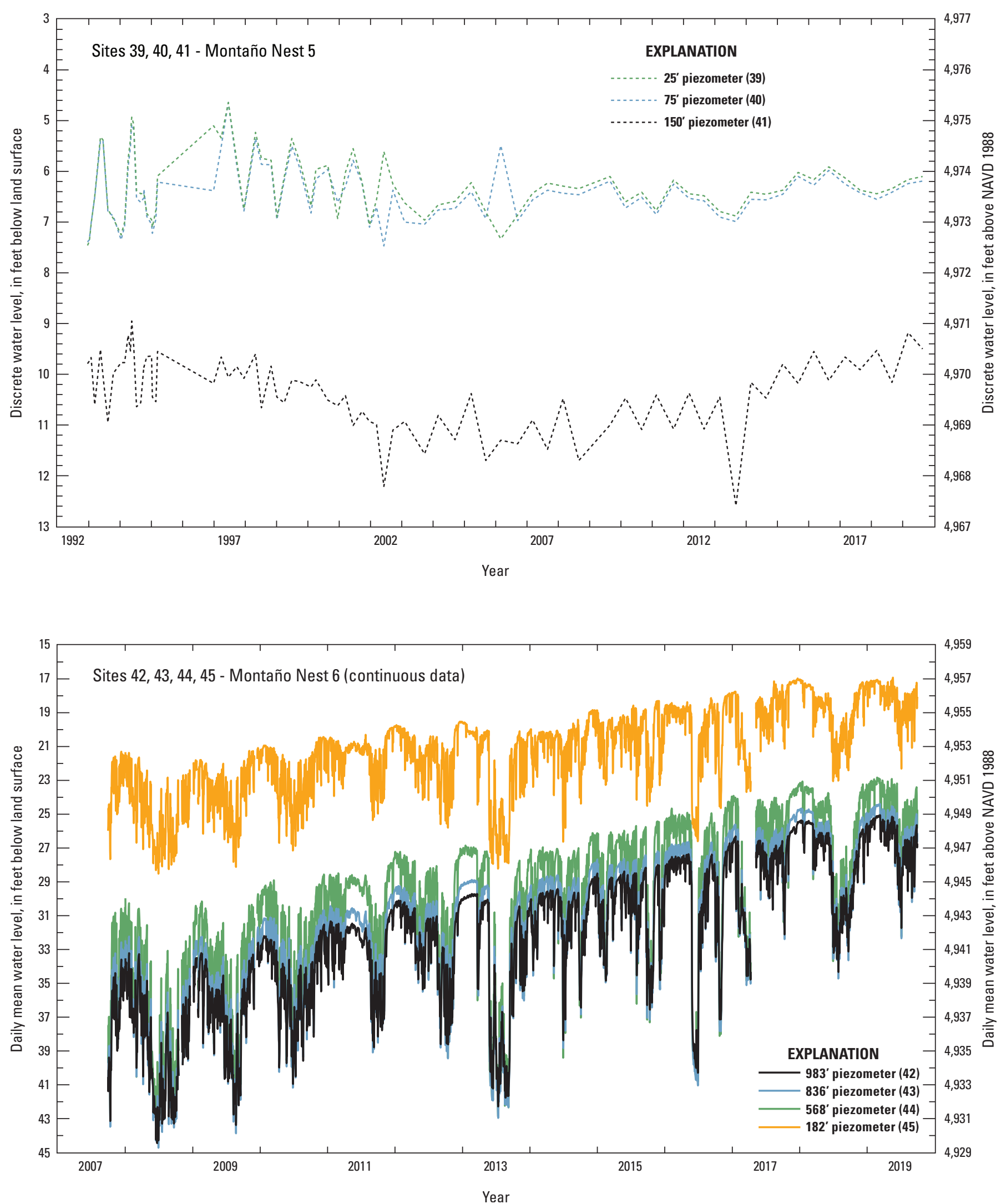

Figure 4. Water-level data for selected wells and piezometers in and adjacent to the Albuquerque Basin, central New Mexico, period of record through September 30, 2019 (NAVD 1988, North American Vertical Datum of 1988). Site numbers correspond to those in table 1.-Continued 

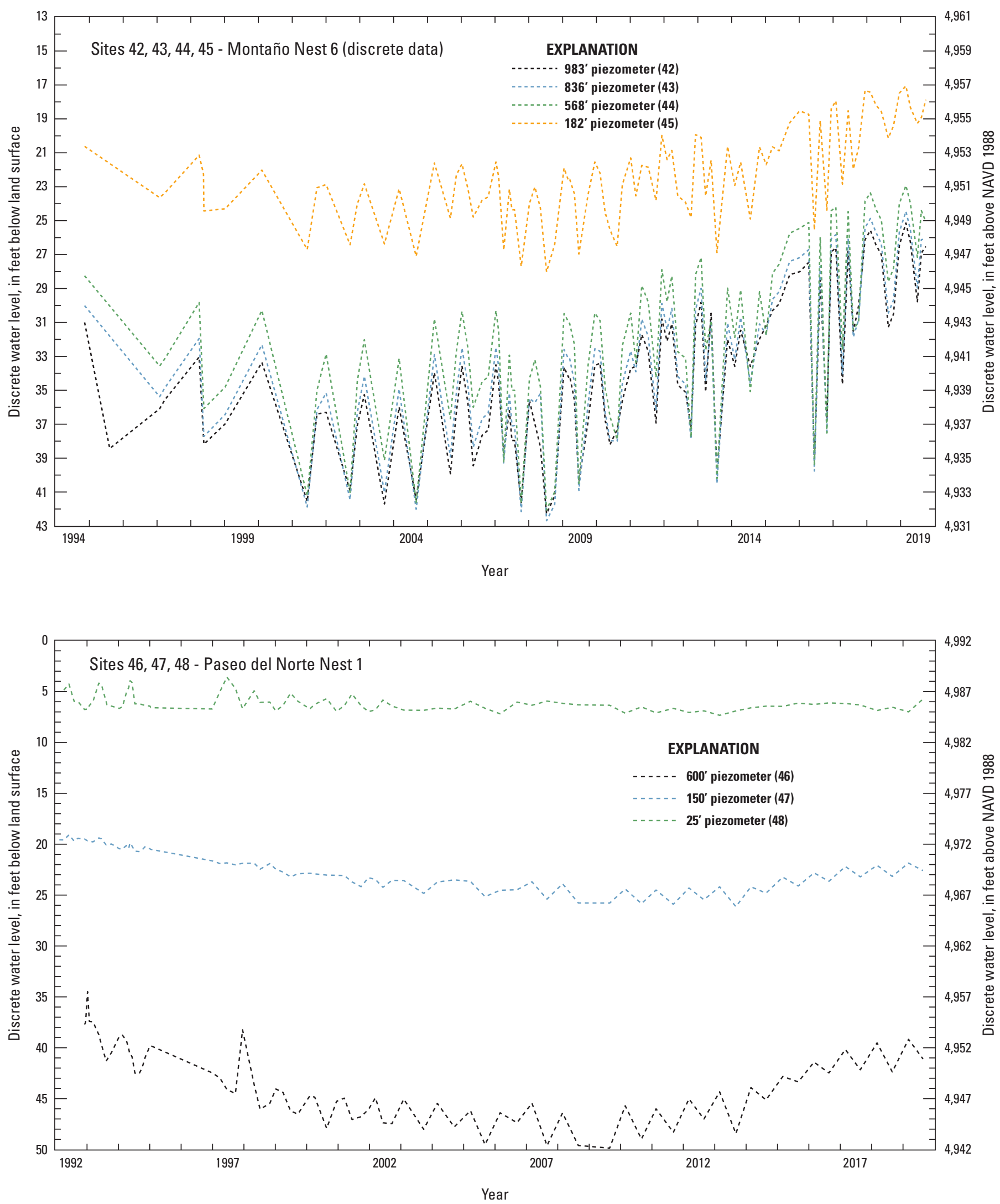

Figure 4. Water-level data for selected wells and piezometers in and adjacent to the Albuquerque Basin, central New Mexico, period of record through September 30, 2019 (NAVD 1988, North American Vertical Datum of 1988). Site numbers correspond to those in table 1.-Continued 

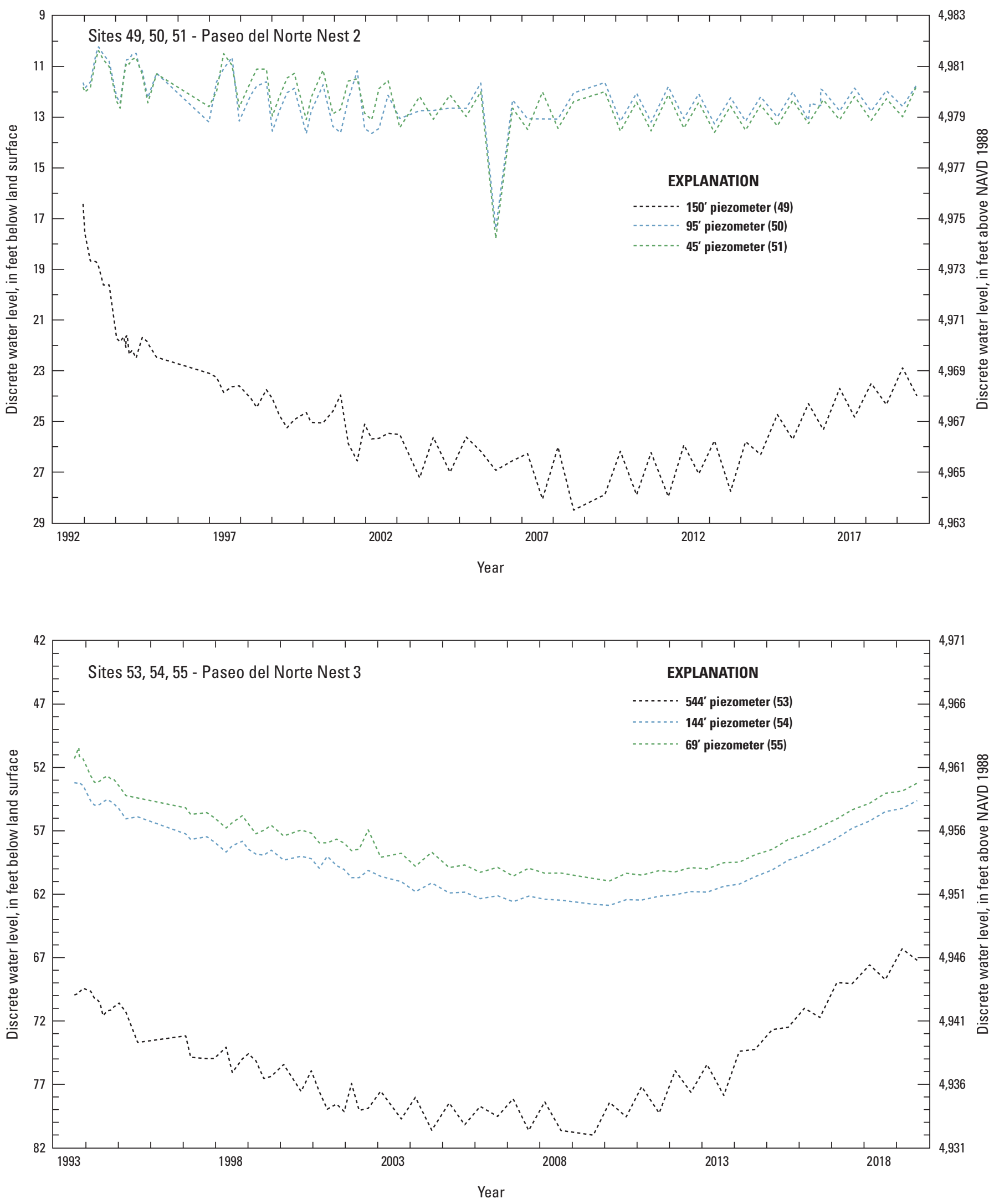

Figure 4. Water-level data for selected wells and piezometers in and adjacent to the Albuquerque Basin, central New Mexico, period of record through September 30, 2019 (NAVD 1988, North American Vertical Datum of 1988). Site numbers correspond to those in table 1.-Continued 

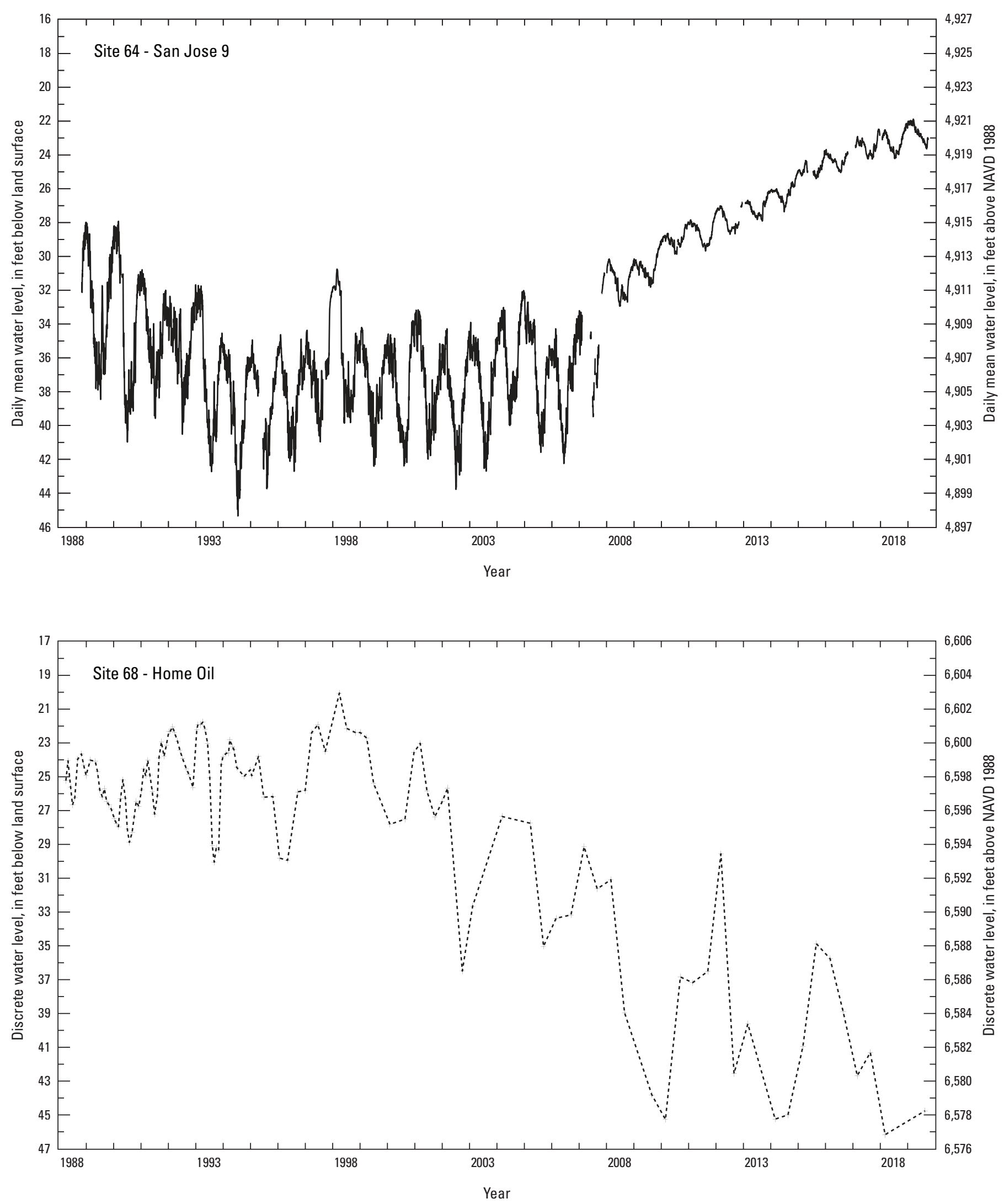

Figure 4. Water-level data for selected wells and piezometers in and adjacent to the Albuquerque Basin, central New Mexico, period of record through September 30, 2019 (NAVD 1988, North American Vertical Datum of 1988). Site numbers correspond to those in table 1.-Continued 

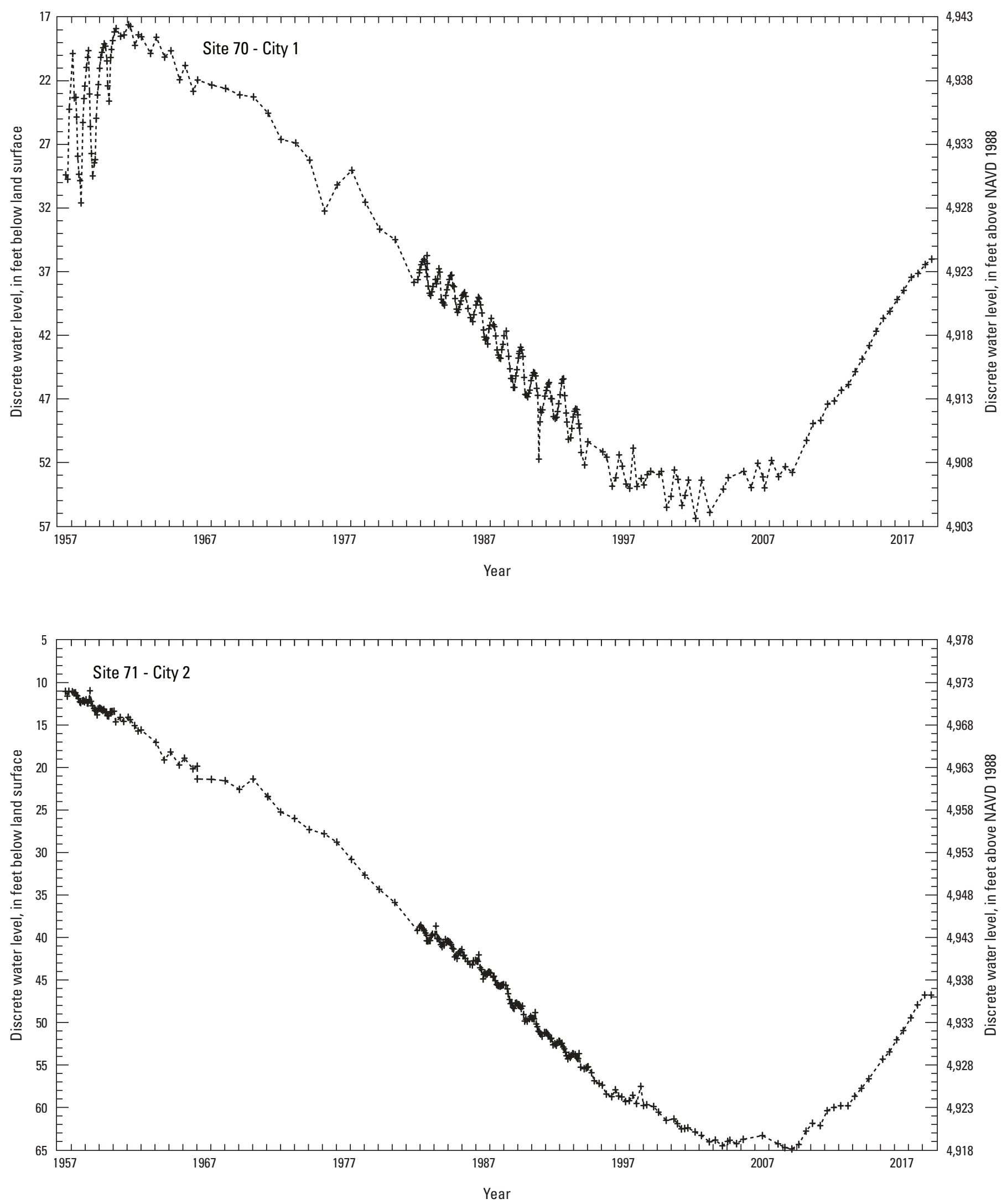

Figure 4. Water-level data for selected wells and piezometers in and adjacent to the Albuquerque Basin, central New Mexico, period of record through September 30, 2019 (NAVD 1988, North American Vertical Datum of 1988). Site numbers correspond to those in table 1.-Continued 

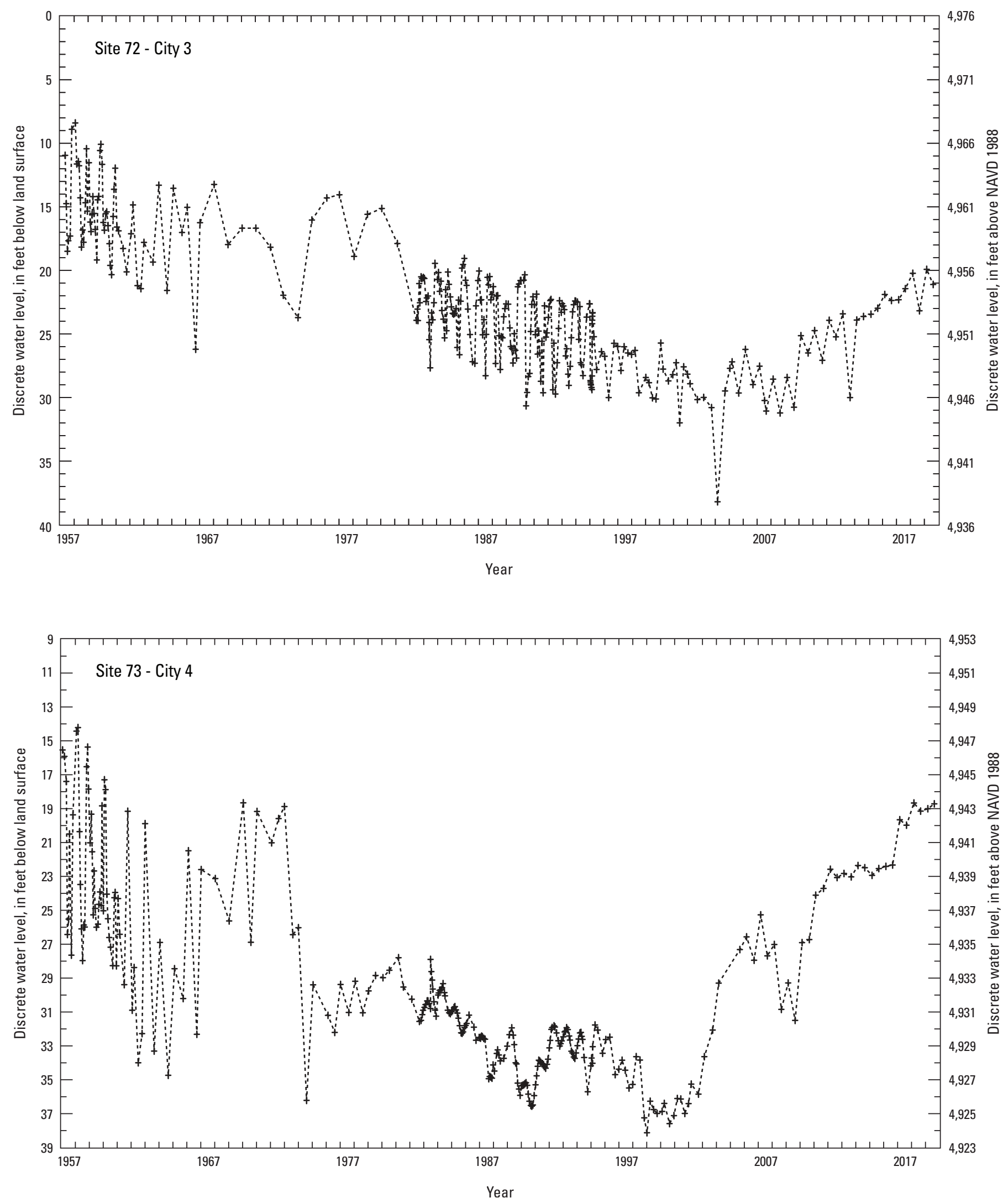

Figure 4. Water-level data for selected wells and piezometers in and adjacent to the Albuquerque Basin, central New Mexico, period of record through September 30, 2019 (NAVD 1988, North American Vertical Datum of 1988). Site numbers correspond to those in table 1.-Continued 

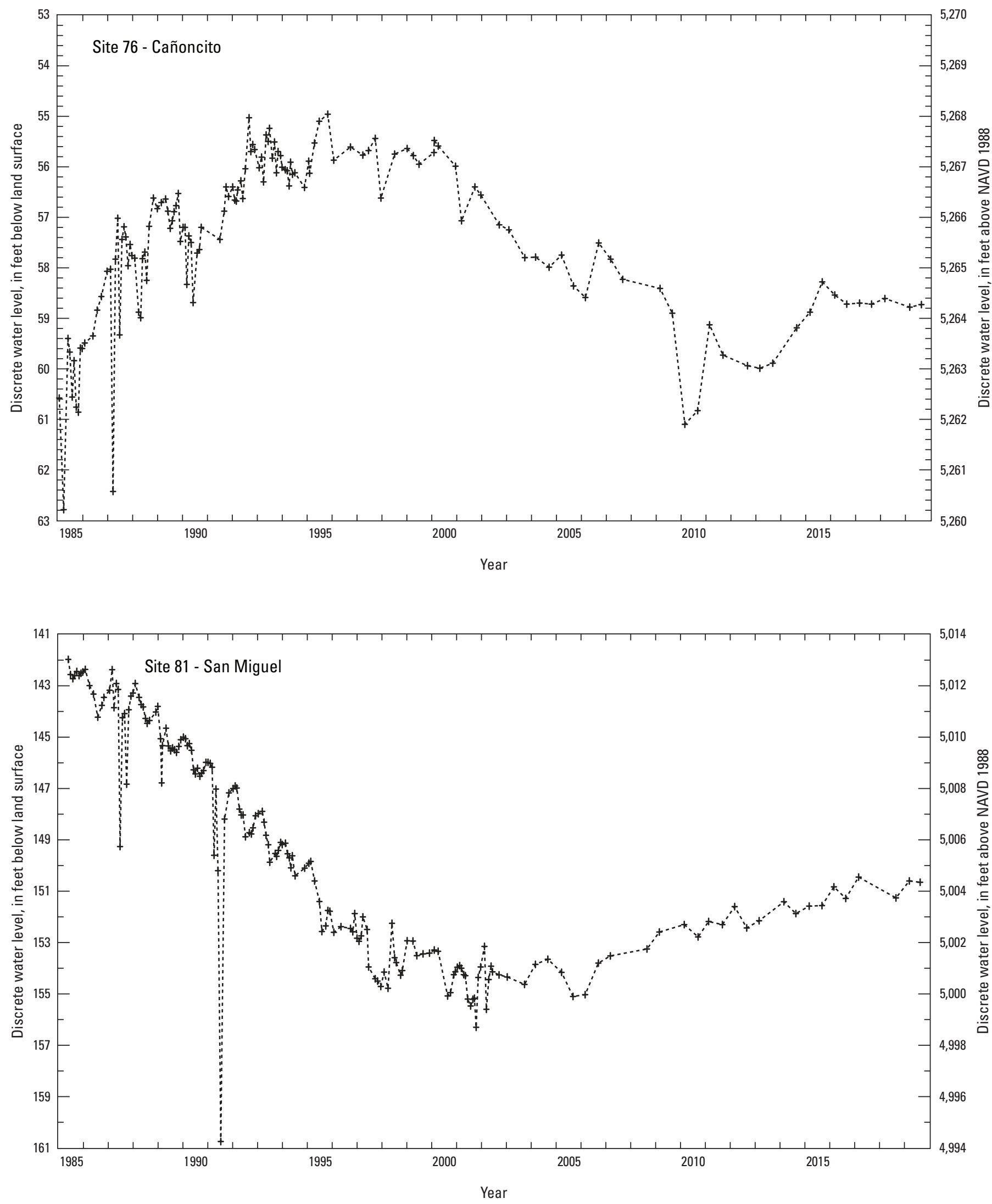

Figure 4. Water-level data for selected wells and piezometers in and adjacent to the Albuquerque Basin, central New Mexico, period of record through September 30, 2019 (NAVD 1988, North American Vertical Datum of 1988). Site numbers correspond to those in table 1.-Continued 

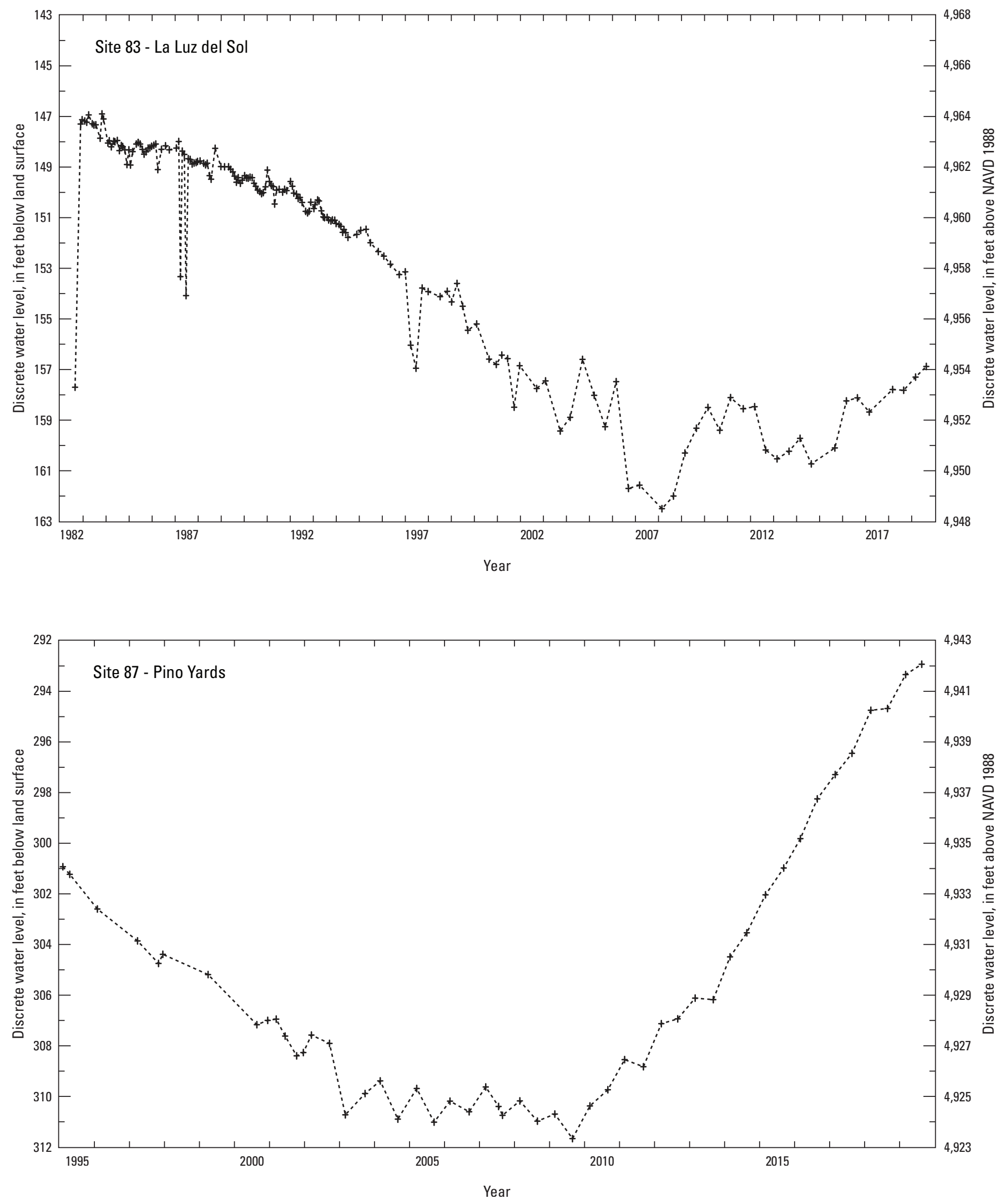

Figure 4. Water-level data for selected wells and piezometers in and adjacent to the Albuquerque Basin, central New Mexico, period of record through September 30, 2019 (NAVD 1988, North American Vertical Datum of 1988). Site numbers correspond to those in table 1.-Continued 

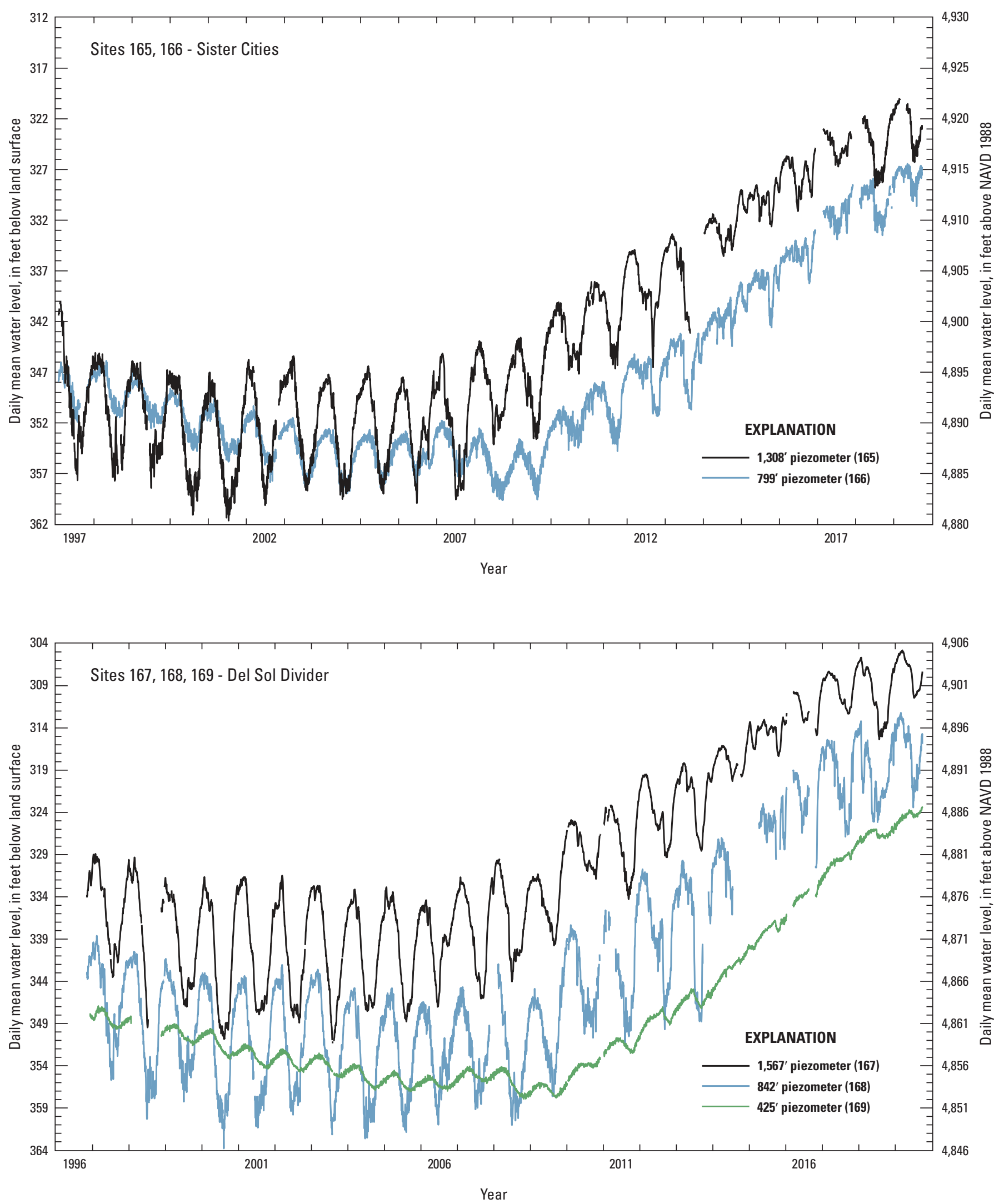

Figure 4. Water-level data for selected wells and piezometers in and adjacent to the Albuquerque Basin, central New Mexico, period of record through September 30, 2019 (NAVD 1988, North American Vertical Datum of 1988). Site numbers correspond to those in table 1.-Continued 

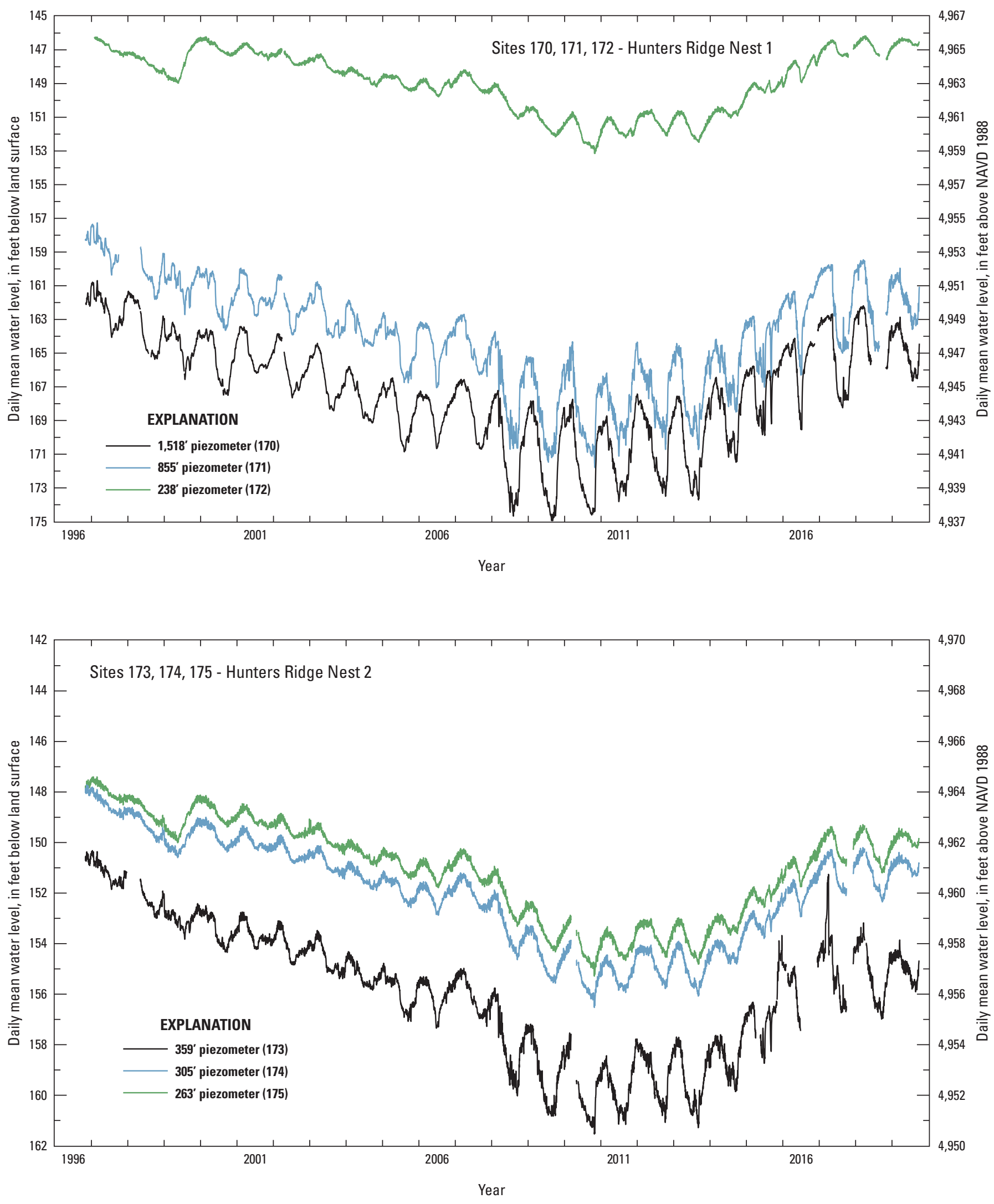

Figure 4. Water-level data for selected wells and piezometers in and adjacent to the Albuquerque Basin, central New Mexico, period of record through September 30, 2019 (NAVD 1988, North American Vertical Datum of 1988). Site numbers correspond to those in table 1.-Continued 

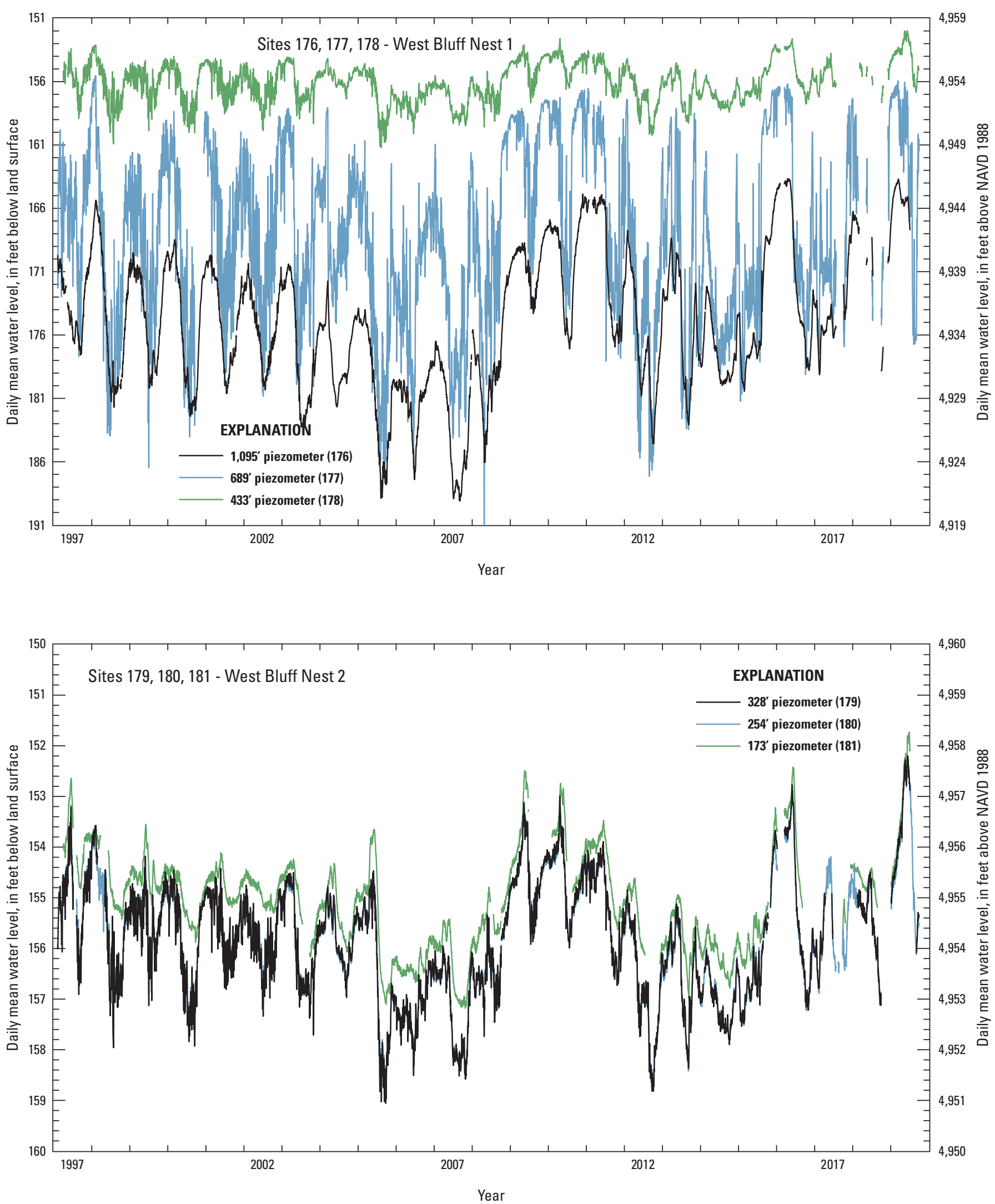

Figure 4. Water-level data for selected wells and piezometers in and adjacent to the Albuquerque Basin, central New Mexico, period of record through September 30, 2019 (NAVD 1988, North American Vertical Datum of 1988). Site numbers correspond to those in table 1.-Continued 

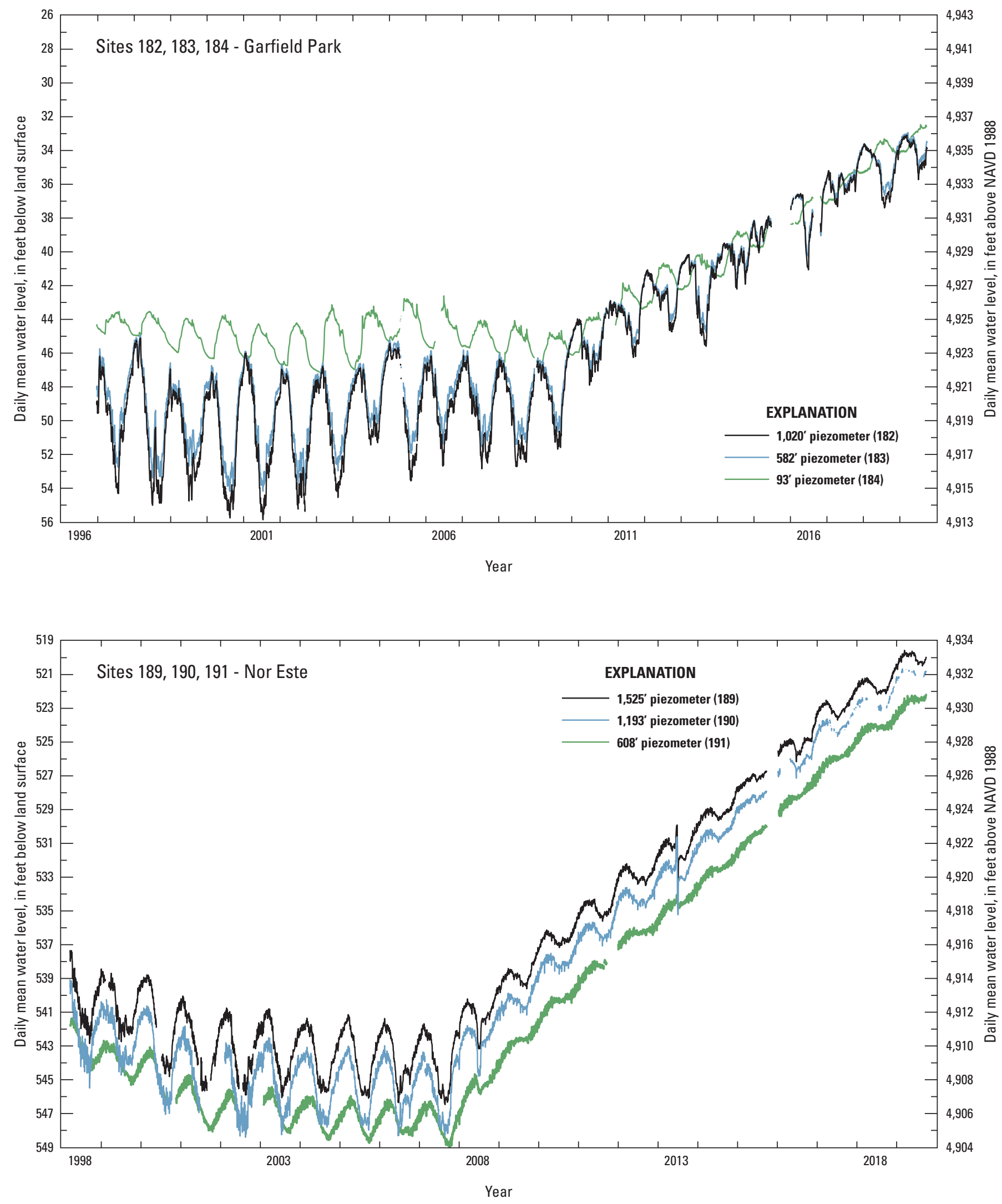

Figure 4. Water-level data for selected wells and piezometers in and adjacent to the Albuquerque Basin, central New Mexico, period of record through September 30, 2019 (NAVD 1988, North American Vertical Datum of 1988). Site numbers correspond to those in table 1.-Continued 

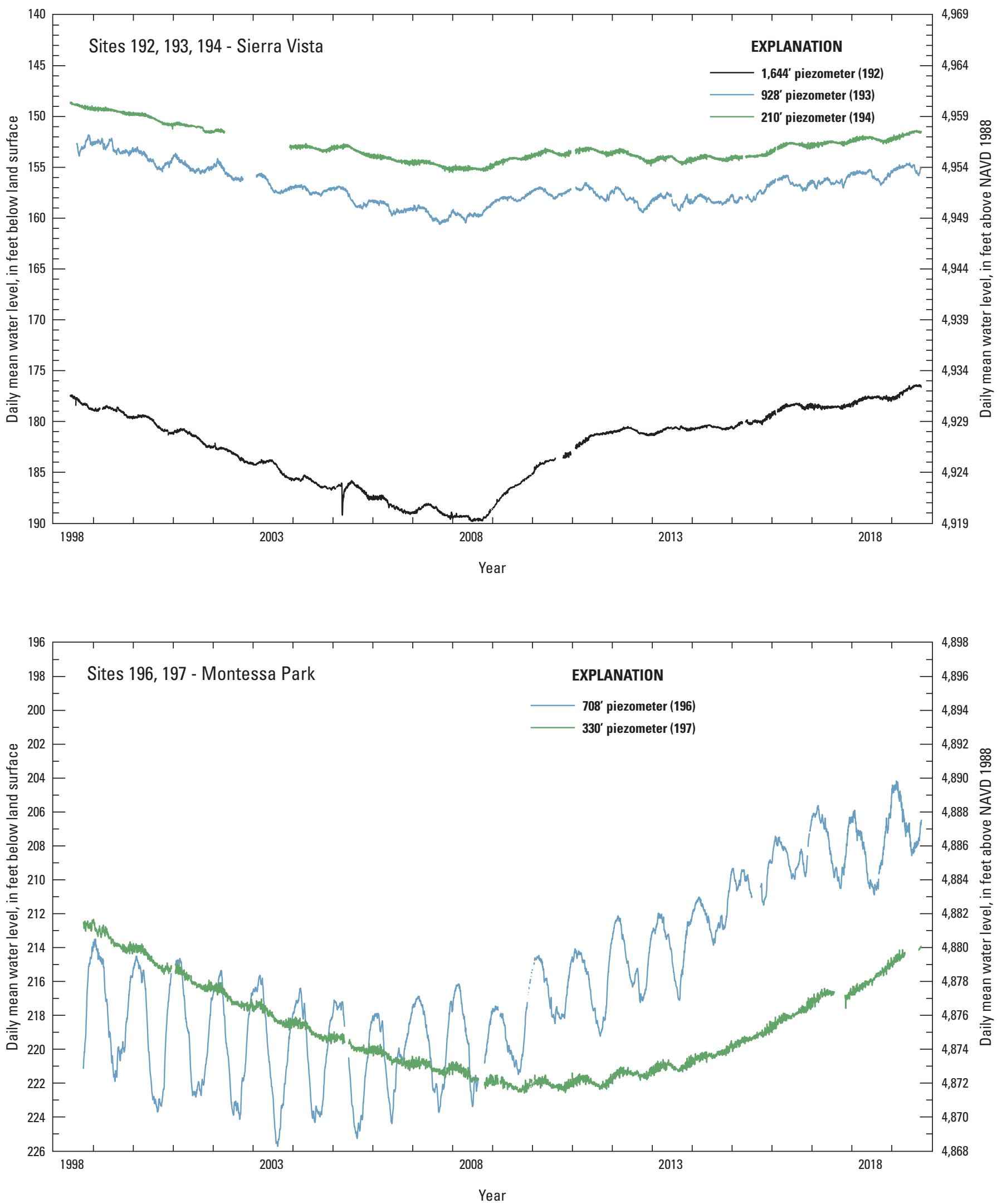

Figure 4. Water-level data for selected wells and piezometers in and adjacent to the Albuquerque Basin, central New Mexico, period of record through September 30, 2019 (NAVD 1988, North American Vertical Datum of 1988). Site numbers correspond to those in table 1.-Continued 

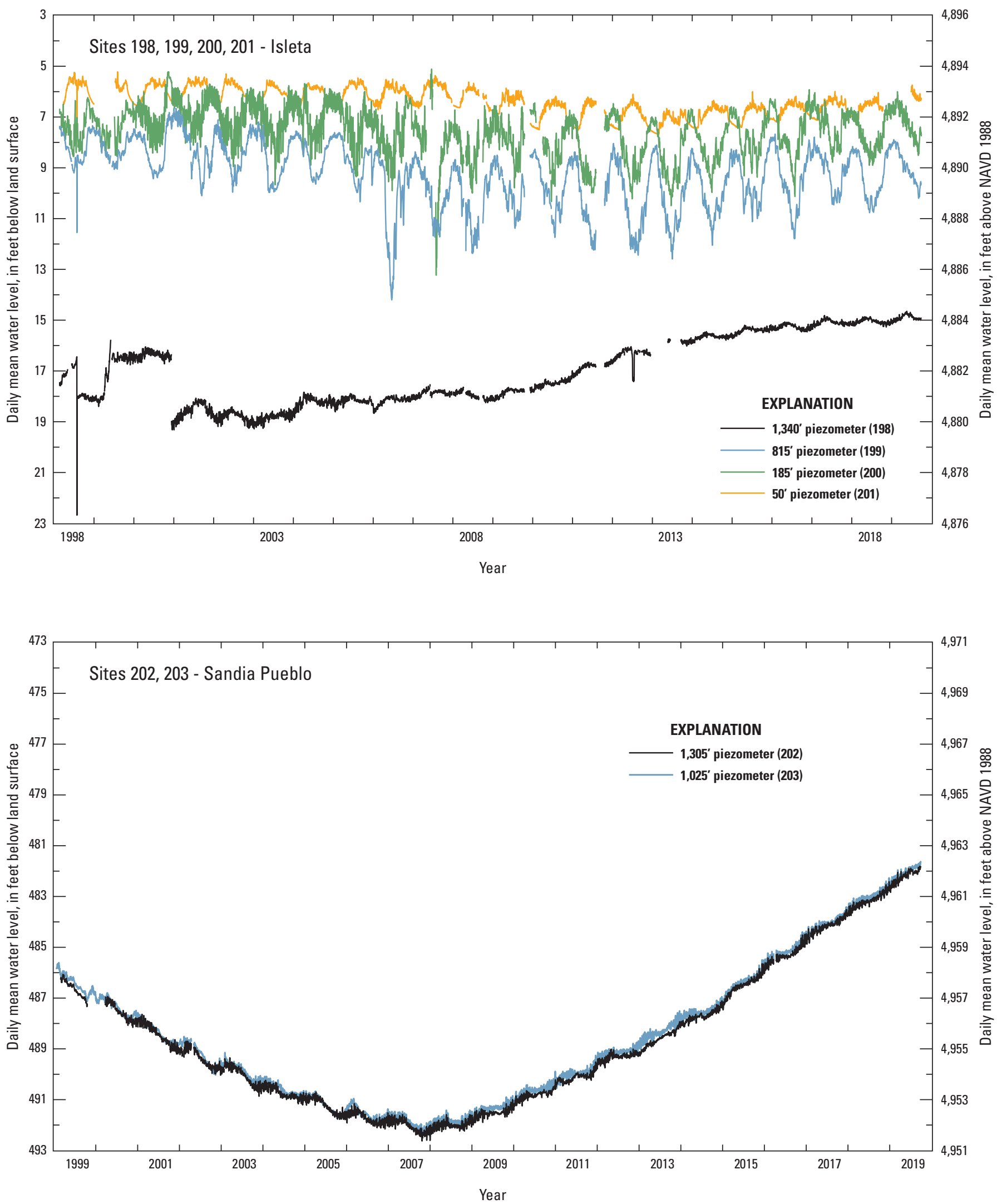

Figure 4. Water-level data for selected wells and piezometers in and adjacent to the Albuquerque Basin, central New Mexico, period of record through September 30, 2019 (NAVD 1988, North American Vertical Datum of 1988). Site numbers correspond to those in table 1.-Continued 

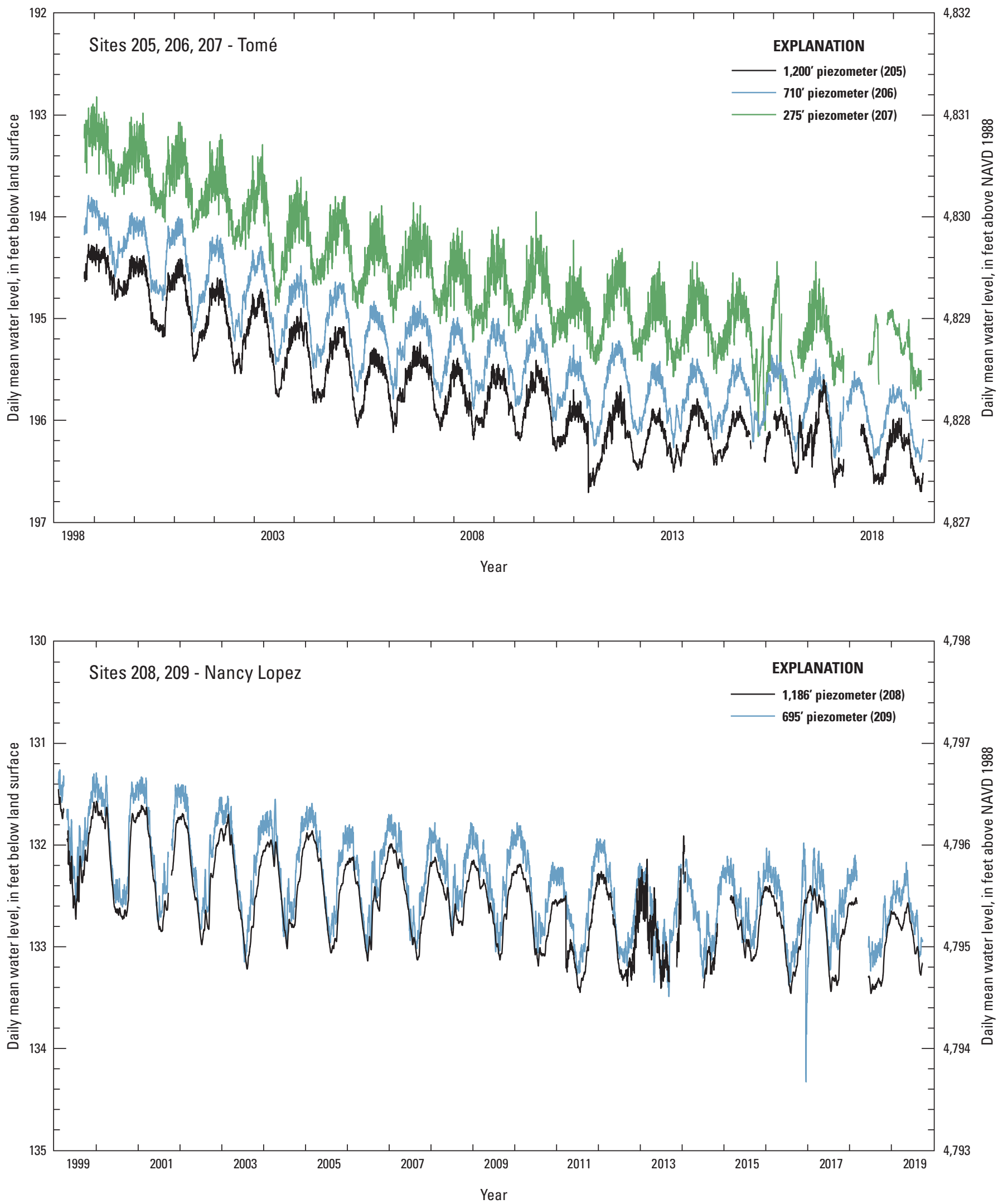

Figure 4. Water-level data for selected wells and piezometers in and adjacent to the Albuquerque Basin, central New Mexico, period of record through September 30, 2019 (NAVD 1988, North American Vertical Datum of 1988). Site numbers correspond to those in table 1.-Continued 

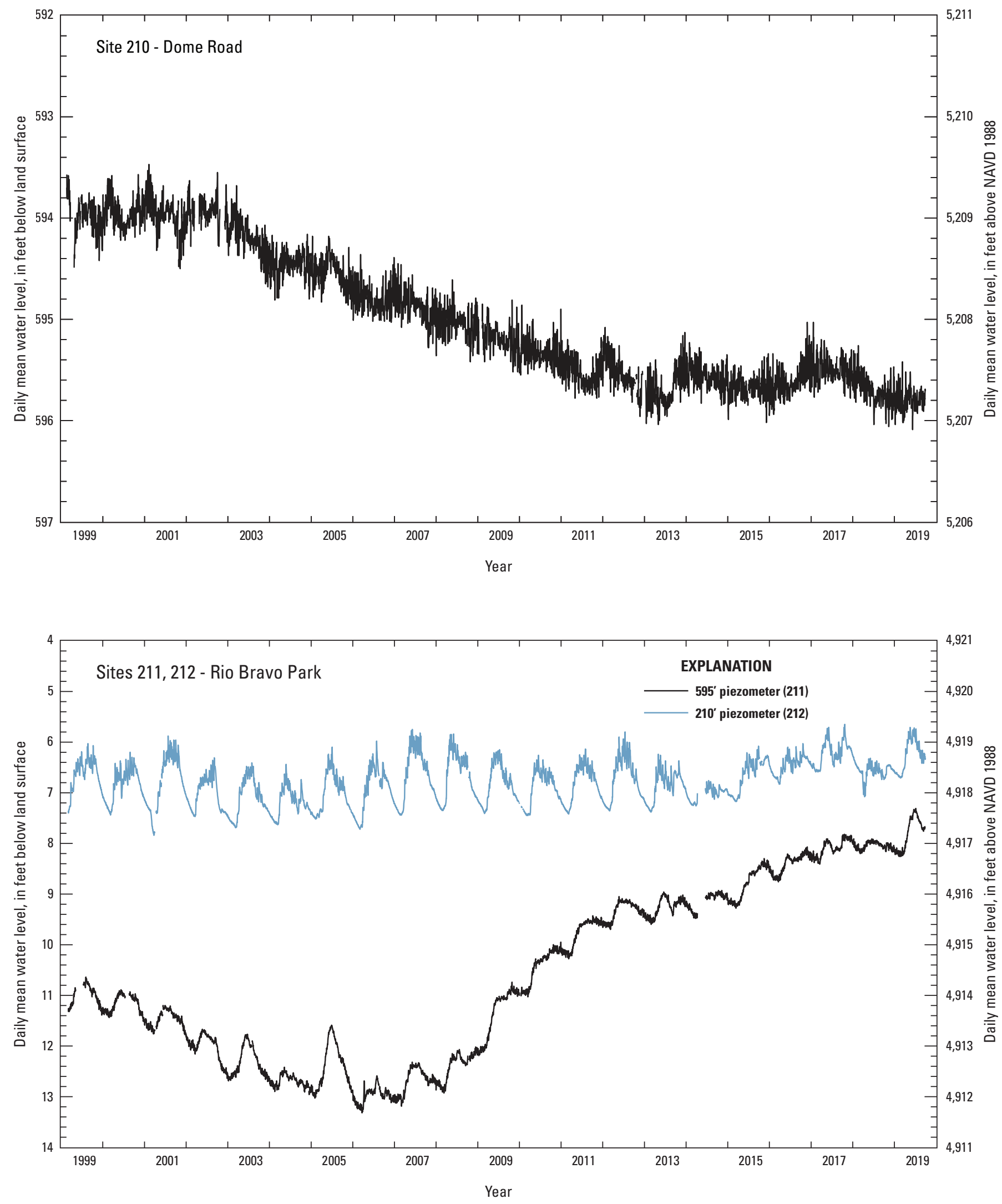

Figure 4. Water-level data for selected wells and piezometers in and adjacent to the Albuquerque Basin, central New Mexico, period of record through September 30, 2019 (NAVD 1988, North American Vertical Datum of 1988). Site numbers correspond to those in table 1.-Continued 

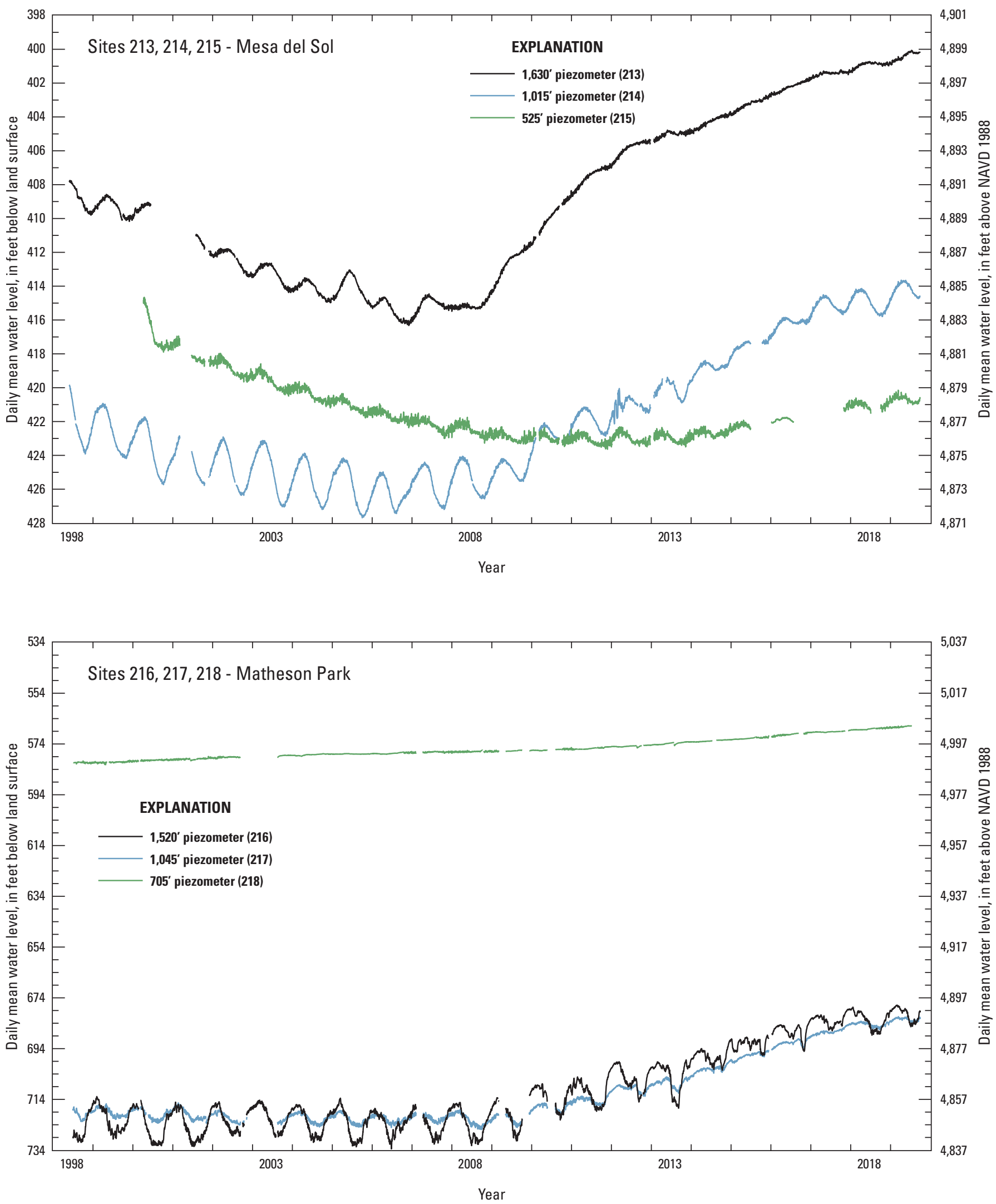

Figure 4. Water-level data for selected wells and piezometers in and adjacent to the Albuquerque Basin, central New Mexico, period of record through September 30, 2019 (NAVD 1988, North American Vertical Datum of 1988). Site numbers correspond to those in table 1.-Continued 

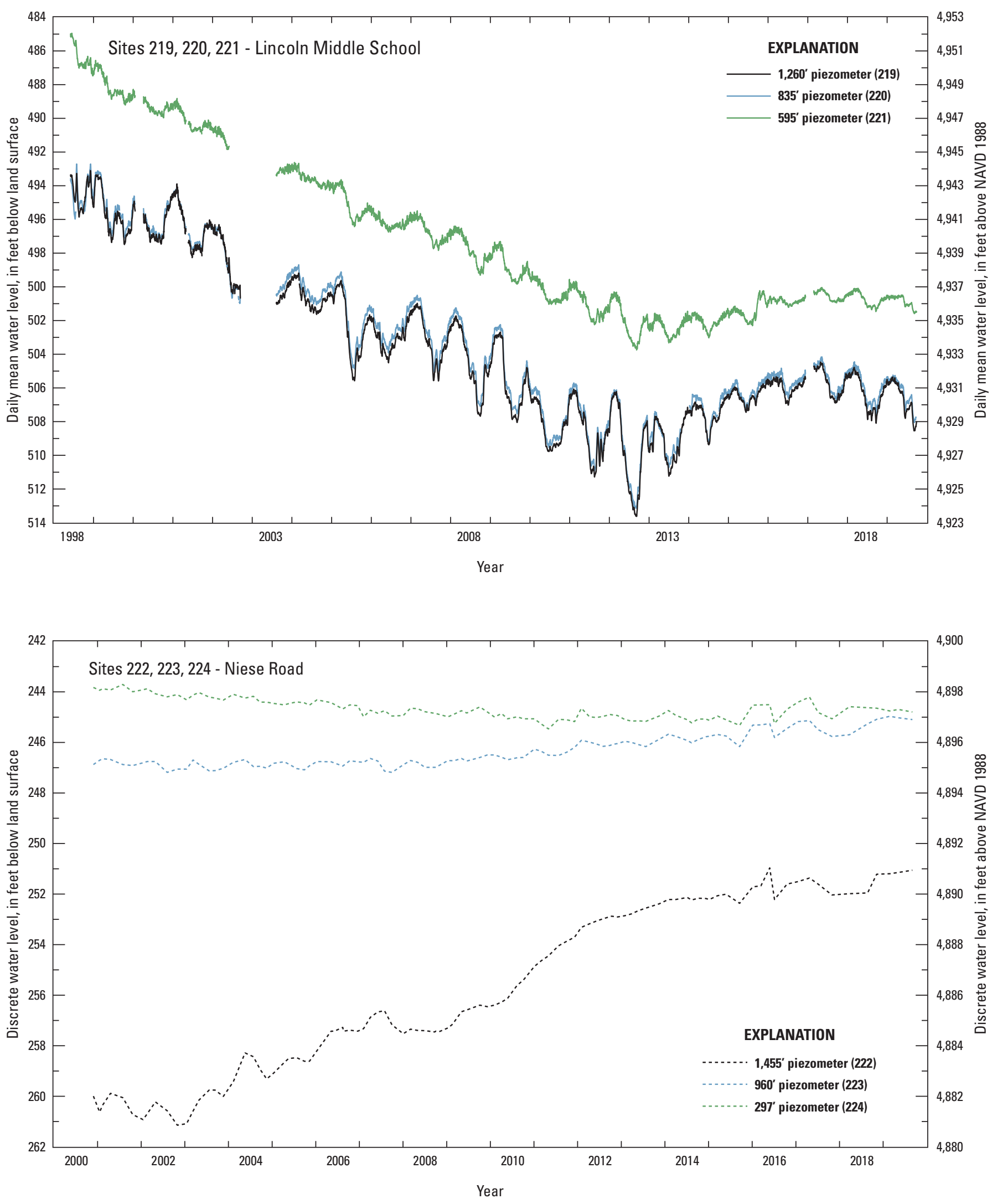

Figure 4. Water-level data for selected wells and piezometers in and adjacent to the Albuquerque Basin, central New Mexico, period of record through September 30, 2019 (NAVD 1988, North American Vertical Datum of 1988). Site numbers correspond to those in table 1.-Continued 

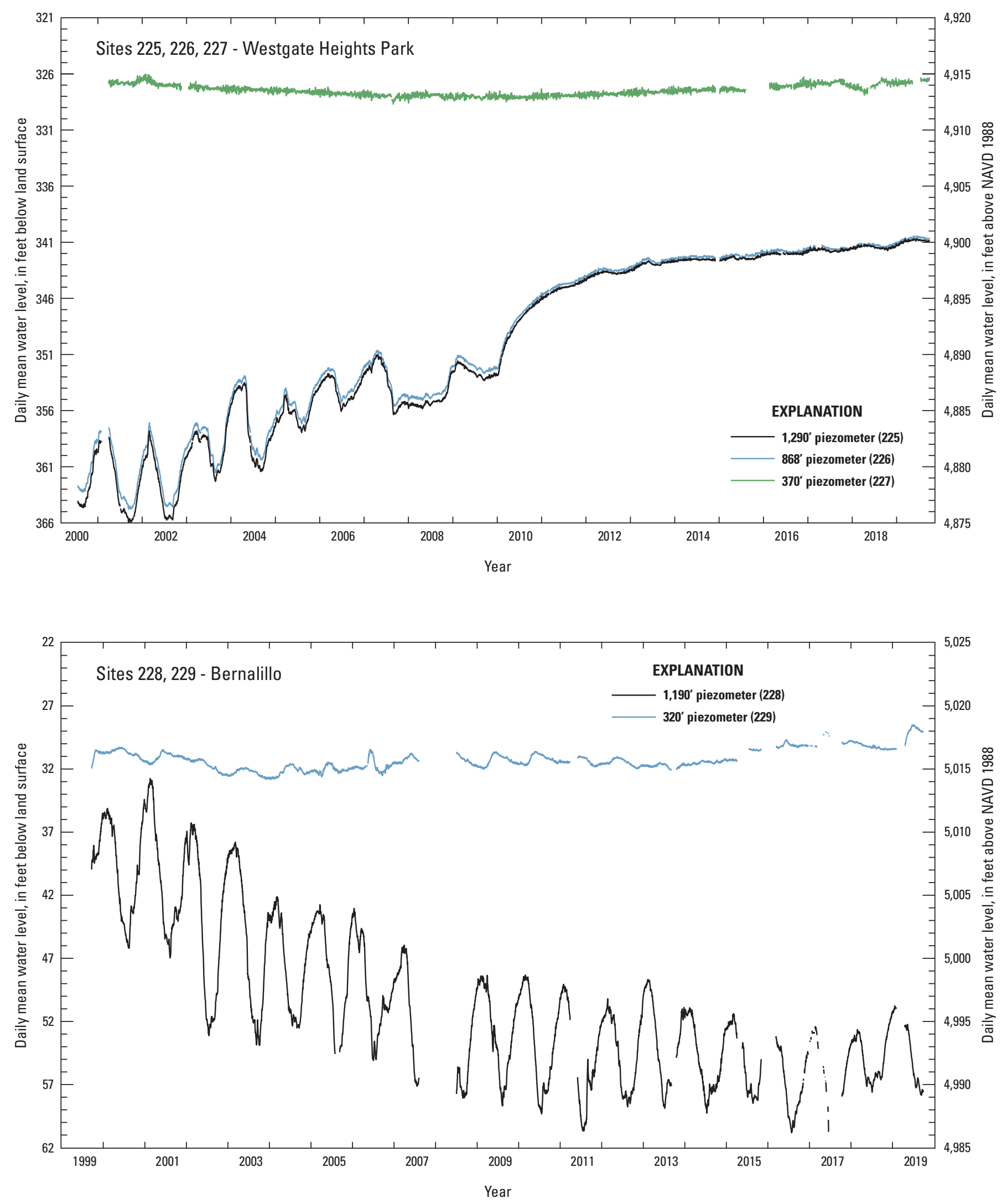

Figure 4. Water-level data for selected wells and piezometers in and adjacent to the Albuquerque Basin, central New Mexico, period of record through September 30, 2019 (NAVD 1988, North American Vertical Datum of 1988). Site numbers correspond to those in table 1.-Continued 

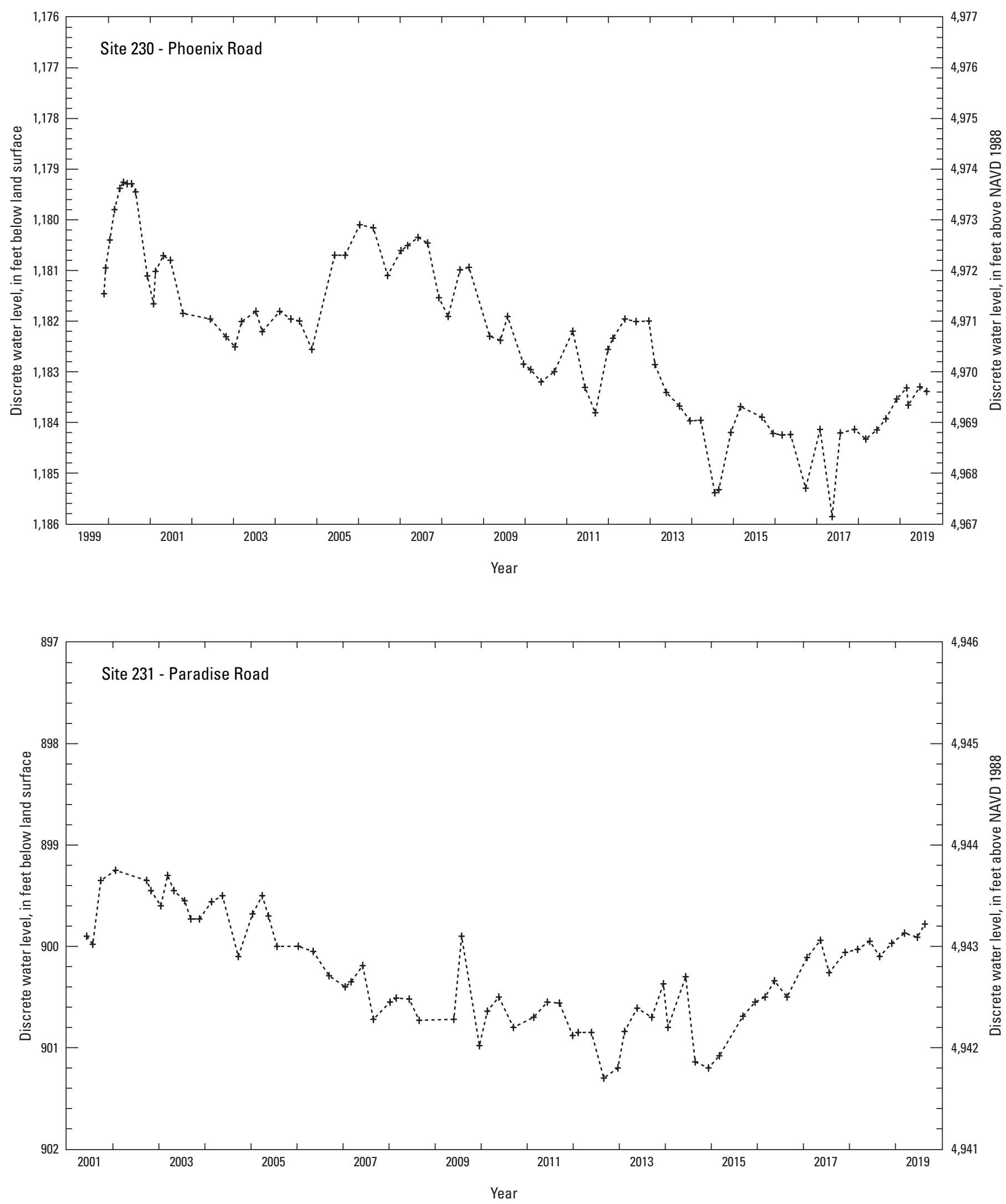

Figure 4. Water-level data for selected wells and piezometers in and adjacent to the Albuquerque Basin, central New Mexico, period of record through September 30, 2019 (NAVD 1988, North American Vertical Datum of 1988). Site numbers correspond to those in table 1.-Continued 

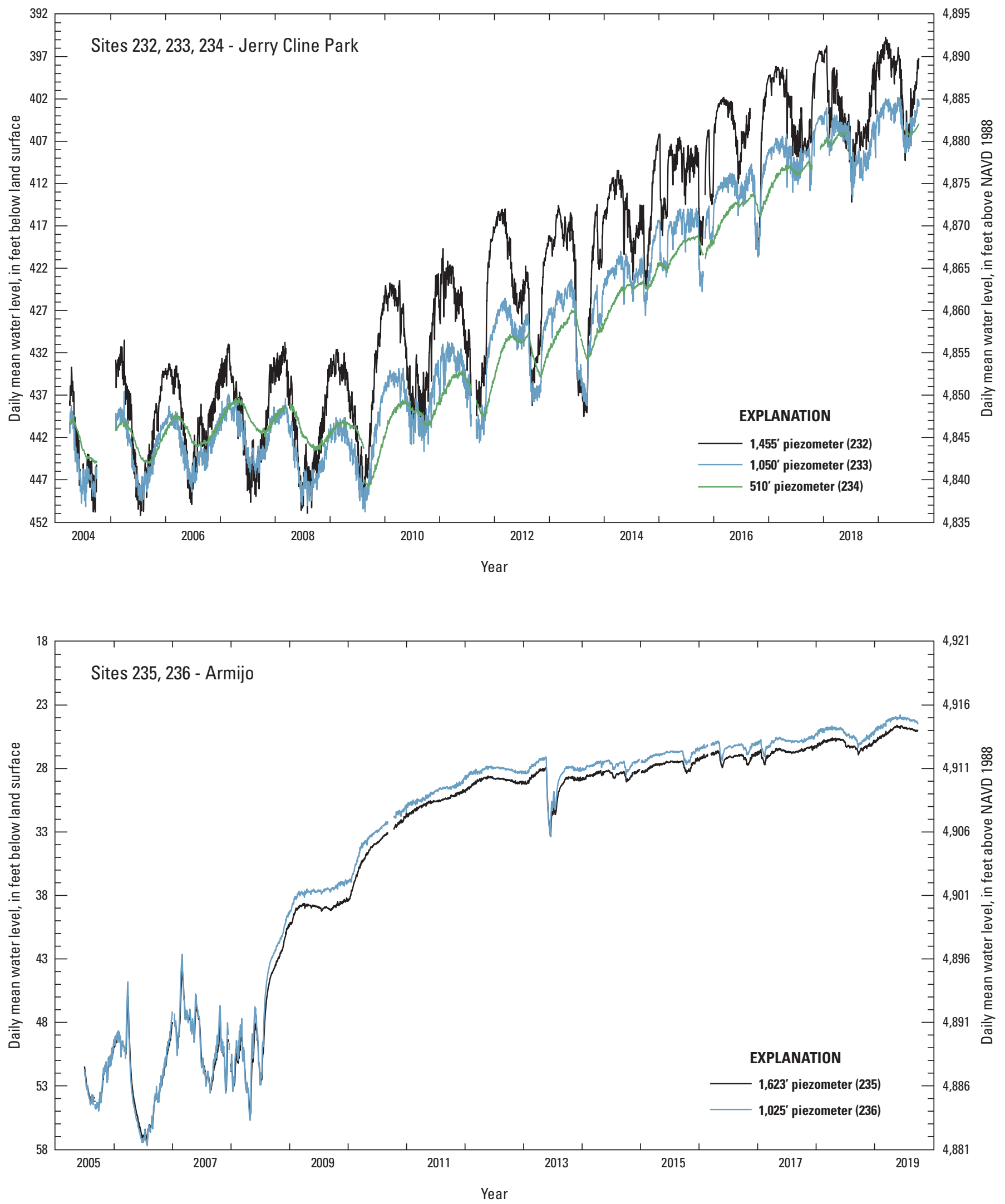

Figure 4. Water-level data for selected wells and piezometers in and adjacent to the Albuquerque Basin, central New Mexico, period of record through September 30, 2019 (NAVD 1988, North American Vertical Datum of 1988). Site numbers correspond to those in table 1.-Continued 


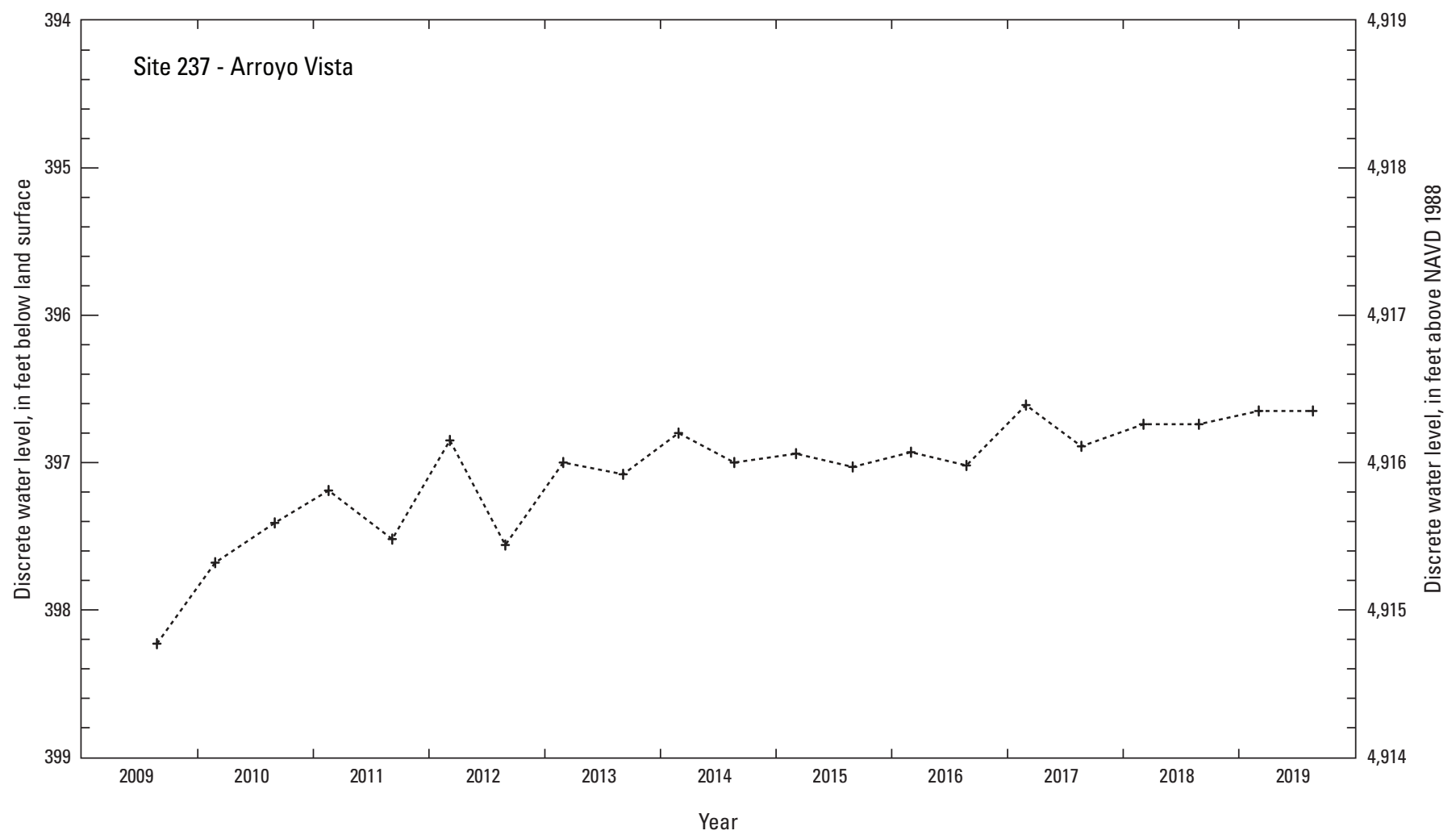

Figure 4. Water-level data for selected wells and piezometers in and adjacent to the Albuquerque Basin, central New Mexico, period of record through September 30, 2019 (NAVD 1988, North American Vertical Datum of 1988). Site numbers correspond to those in table 1.-Continued 


\section{Acknowledgments}

The author would like to thank Andre Ritchie for developing the method for producing the graphs in figure 4 . The author would also like to thank the many USGS staff who have contributed to collecting the water-level measurements used in this report, notably Christina F. Bryant, Harold Nelson, and Elaiya Jurney.

\section{References Cited}

Bartolino, J.R., and Cole, J.C., 2002, Ground-water resources of the Middle Rio Grande Basin, New Mexico: U.S. Geological Survey Circular 1222, 132 p. [Also available at http://pubs.usgs.gov/circ/2002/circ1222/.]

Beman, J.E., 2007, Water-level data for the Albuquerque Basin and adjacent areas, central New Mexico, period of record through September 30, 2006: U.S. Geological Survey OpenFile Report 2007-1273, 34 p. [Also available at http://pubs. usgs.gov/of/2007/1273/.]

Beman, J.E., 2008, Water-level data for the Albuquerque Basin and adjacent areas, central New Mexico, period of record through September 30, 2007: U.S. Geological Survey OpenFile Report 2008-1255, 32 p. [Also available at http://pubs. usgs.gov/of/2008/1255/.]

Beman, J.E., 2009, Water-level data for the Albuquerque Basin and adjacent areas, central New Mexico, period of record through September 30, 2008: U.S. Geological Survey OpenFile Report 2009-1125, 37 p. [Also available at http://pubs. usgs.gov/of/2009/1125/.]

Beman, J.E., 2011, Water-level data for the Albuquerque Basin and adjacent areas, central New Mexico, period of record through September 30, 2010: U.S. Geological Survey Data Series 623, 28 p. [Also available at http://pubs.usgs.gov/ ds/623/.]

Beman, J.E., 2012, Water-level data for the Albuquerque Basin and adjacent areas, central New Mexico, period of record through September 30, 2011: U.S. Geological Survey Data Series 714, 29 p. [Also available at http://pubs.usgs.gov/ ds/714/.]

Beman, J.E., 2013, Water-level data for the Albuquerque Basin and adjacent areas, central New Mexico, period of record through September 30, 2012: U.S. Geological Survey Data Series 790, 28 p. [Also available at https://doi.org/10.3133/ ds790.]
Beman, J.E., 2014, Water-level data for the Albuquerque Basin and adjacent areas, central New Mexico, period of record through September 30, 2013: U.S. Geological Survey Data Series 873, 40 p. [Also available at https://doi.org/10.3133/ ds873.]

Beman, J.E., 2015, Water-level data for the Albuquerque Basin and adjacent areas, central New Mexico, period of record through September 30, 2014: U.S. Geological Survey Data Series 963, 42 p. [Also available at https://doi.org/10.3133/ ds963.]

Beman, J.E., and Bryant, C.F., 2016, Water-level data for the Albuquerque Basin and adjacent areas, central New Mexico, period of record through September 30, 2015: U.S. Geological Survey Data Series 1025, 39 p. [Also available at https://doi.org/10.3133/ds1025.]

Beman, J.E., Ritchie, A.B., and Galanter, A.E., 2019, Waterlevel data for the Albuquerque Basin and adjacent areas, central New Mexico, period of record through September 30, 2017: U.S. Geological Survey Data Series 1113, 39 p. [Also available at https://doi.org/10.3133/ds1113.]

Beman, J.E., and Torres, L.T., 2010, Water-level data for the Albuquerque Basin and adjacent areas, central New Mexico, period of record through September 30, 2009: U.S. Geological Survey Open-File Report 2010-1228, 31 p. [Also available at http://pubs.usgs.gov/of/2010/1228/.]

Bureau of Land Management, 2009, Manual of surveying instructions for the survey of the public lands of the United States: U.S. Department of the Interior, Bureau of Land Management Cadastral Survey, 513 p., [Also available at https://edit.blm.gov/sites/blm.gov/files/Manual_Of_ Surveying_Instructions_2009.pdf.]

Cunningham, W.L., and Schalk, C.W., comps., 2011, Groundwater technical procedures of the U.S. Geological Survey: U.S. Geological Survey Techniques and Methods 1-A1, 151 p., accessed September 30, 2014, at http://pubs. usgs.gov/tm/1a1/.

DeWees, R.K., 2001, Water-level data for the Albuquerque Basin and adjacent areas, central New Mexico, period of record through 2000: U.S. Geological Survey Open-File Report 2001-184, 62 p. [Also available at http://pubs. er.usgs.gov/publication/ofr01184.]

DeWees, R.K., 2002, Water-level data for the Albuquerque Basin and adjacent areas, central New Mexico, period of record through 2001: U.S. Geological Survey Open-File Report 2002-312, 41 p. [Also available at http://pubs. er.usgs.gov/publication/ofr02312.] 
DeWees, R.K., 2003, Water-level data for the Albuquerque Basin and adjacent areas, central New Mexico, period of record through 2002: U.S. Geological Survey Open-File Report 2003-321, 41 p. [Also available at http://pubs. er.usgs.gov/publication/ofr03321.]

DeWees, R.K., 2006, Water-level data for the Albuquerque Basin and adjacent areas, central New Mexico, period of record through 2004: U.S. Geological Survey Open-File Report 2006-1281, 40 p. [Also available at http://pubs.usgs. gov/of/2006/1281/.]

Kues, G.E., 1987, Ground-water-level data for the Albuquerque-Belen Basin, New Mexico, through water year 1985: U.S. Geological Survey Open-File Report 87-116, 51 p. [Also available at http://pubs.er.usgs.gov/publication/ ofr87116.]

Rankin, D.R., 1994, Water-level data for the Albuquerque Basin, New Mexico, October 1, 1986, through September 30, 1990: U.S. Geological Survey Open-File Report 94-349, 29 p. [Also available at http://pubs.er.usgs.gov/ publication/ofr94349.]

Rankin, D.R., 1996, Water-level data for the Albuquerque Basin, New Mexico, period of record through September 30, 1995: U.S. Geological Survey Open-File Report 96-664A, 28 p. [Also available at http://pubs.er.usgs.gov/ publication/ofr96664A.]

Rankin, D.R., 1998, Water-level data for the Albuquerque Basin, central New Mexico, period of record through 1997: U.S. Geological Survey Open-File Report 98-408, 28 p. [Also available at http://pubs.er.usgs.gov/publication/ ofr98408.]

Rankin, D.R., 1999, Water-level data for the Albuquerque Basin and adjacent areas, New Mexico, period of record through 1998: U.S. Geological Survey Open-File Report 99-269, 27 p. [Also available at http://pubs.er.usgs.gov/ publication/ofr99269.]
Rankin, D.R., 2000, Water-level data for the Albuquerque Basin and adjacent areas, central New Mexico, period of record through 1999: U.S. Geological Survey Open-File Report 2000-231, 62 p. [Also available at http://pubs. er.usgs.gov/publication/ofr00231.]

Ritchie, A.B., and Galanter, A.E., 2019, Water-level data for the Albuquerque Basin and adjacent areas, central New Mexico, period of record through September 30, 2018: U.S. Geological Survey Data Series 1116, 40 p. [Also available at https://doi.org/10.3133/ds1116.]

Thorn, C.R., McAda, D.P., and Kernodle, J.M., 1993, Geohydrologic framework and hydrologic conditions in the Albuquerque Basin, central New Mexico: U.S. Geological Survey Water-Resources Investigations Report 93-4149, 106 p. [Also available at http://pubs.er.usgs.gov/publication/ wri934149.]

U.S. Census Bureau, 2012, New Mexico-2010 census of population and housing, population and housing unit counts: Washington, D.C., U.S. Census Bureau, CPH-2-33, 79 p. [Also available at https://www2.census.gov/library/ publications/decennial/2010/cph-2/cph-2-33.pdf.]

U.S. Census Bureau, 2019, Centers of population for the 2010 census, centers of population by census tract: U.S. Census Bureau database, accessed June 27, 2019, at https://www. census.gov/geographies/reference-files/2010/geo/2010centers-population.html.

U.S. Geological Survey, 2020, USGS water data for the Nation: U.S. Geological Survey National Water Information System database, accessed September 30, 2020, at https:// doi.org/10.5066/F7P55KJN.

For more information about this publication, contact:

Director, New Mexico Water Science Center

U.S. Geological Survey

6700 Edith Blvd. NE

Albuquerque, NM 87113

Publishing support provided by

Lafayette Publishing Service Center 



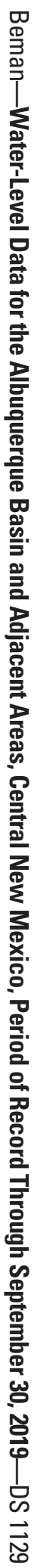

